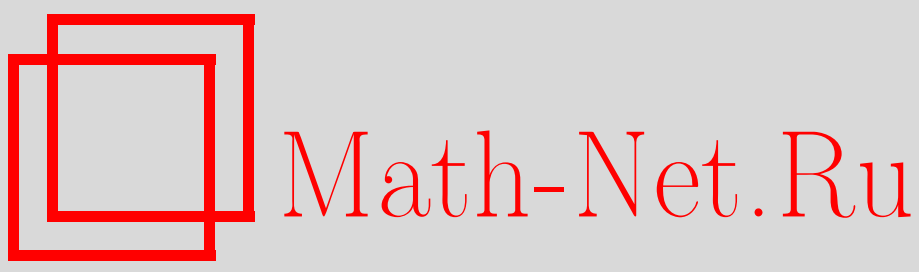

А. Ю. Пилипенко, Преобразования мер в бесконечномерных пространствах потоком, порожденным стохастическим дифференциальным уравнением, Матем. сб., 2003, том 194, номер 4, 85-106

DOI: https://doi.org/10.4213/sm729

Использование Общероссийского математического портала Math-Net.Ru подразумевает, что вы прочитали и согласны с пользовательским соглашением

http://www.mathnet.ru/rus/agreement

Параметры загрузки:

IP : 3.89 .197 .203

26 апреля 2023 г., 14:57:24 
УДК 519.21

\section{А.Ю. Пилипенко}

\section{Преобразования мер в бесконечномерных пространствах потоком, порожденным стохастическим дифференциальным уравнением}

Пусть $\mu$ - гауссовская мера в пространстве $X, H$ - пространство Камерона-Мартина меры $\mu$. Рассмотрим стохастическое дифференциальное уравнение

$$
\begin{gathered}
d \xi(u, t)=a_{t}(\xi(u, t)) d t+\sum_{n} \sigma_{t}^{n}(\xi(u, t)) d \omega_{n}(t), \quad t \in[0, T], \\
\xi(u, 0)=u,
\end{gathered}
$$

где $u \in X, a, \sigma_{n}$ - функции, принимающие значения в $H, \omega_{n}(t), n \geqslant 1,-$ независимые одномерные винеровские процессы. Определим мерозначный случайный процесс $\mu_{t}:=\mu \circ \xi(\cdot, t)^{-1}$. При некоторых естественных условиях на коэффиициенты исходного уравнения доказано, что для почти всех $\omega$ меры $\mu_{t}(\omega)$ эквивалентны $\mu$. Для плотностей Радона-Никодима получены явные выражения.

Библиография: 10 названий.

\section{§1. Введение}

Рассмотрим поток $\xi(u, t), u \in \mathbb{R}^{d}, t \in[0, T]$, порожденный стохастическим дифференциальным уравнением в евклидовом пространстве $\mathbb{R}^{d}$ :

$$
\begin{gathered}
d \xi(u, t)=a_{t}(\xi(u, t)) d t+\sum_{n} \sigma_{t}^{n}(\xi(u, t)) d \omega_{n}(t), \\
\xi(u, 0)=u .
\end{gathered}
$$

Предположим, что мера $\mu$ в $\mathbb{R}^{d}$ имеет гладкую положительную плотность относительно меры Лебега. Х. Кунитой [1] было доказано, что при некоторых естественных условиях на гладкость и ограниченность коэффициентов (1.1) меры $\mu_{t}(\omega):=\mu \circ \xi(\cdot, t, \omega)^{-1}$ абсолютно непрерывны для п.в. $\omega$, и для плотности $\rho_{t}(\omega)(u)=\frac{d \mu_{t}(\omega)}{d \mu}(u), u \in \mathbb{R}^{d}$, имеет место стохастический аналог теоремы Лиувилля.

В настоящей статье рассматривается подобная задача о преобразовании мер потоком, порожденным стохастическим дифференциальным уравнением в бесконечномерном пространстве. Отметим несколько существенных различий между конечномерным и бесконечномерным случаем. Во-первых, даже линейное стохастическое уравнение может не порождать потока в бесконечномерном пространстве [2]. Во-вторых, это связано с отсутствием канонической бесконечномерноймеры Лебега и, соответственно, мер с гладкой плотностью. Естественным обобшением

Работа выполнена при частичной поддержке Министерства образования и науки Украины (грант № 01.07/103). 
мер с гладкой плотностью на бесконечномернњй случай являются дифференцируемые меры. Наиболее интересным и исследуемым классом таких мер являются гауссовские меры, которые будут рассматриваться в нашей статье.

Преобразования мер потоками, порожденными обыкновенными (но не стохастическими) дифференциальными уравнениями в бесконечномерном случае, рассматривались многими авторами, см., например, работы [3]-[6] и ссылки в них.

Основным результатом настоящей статьи является теорема 3.1, в которой получены условия на коэффициенты $a_{t}, \sigma_{t}^{n}$ уравнения $(1.1)$, гарантирующие эквивалентность мер $\mu$ и $\mu_{t}(\omega)$ для п.в. $\omega$ в случае, когда $\mu-$ радоновская гауссовская мера. Для плотностей Радона-Никодима получены явные выражения, совпадающие с соответствующими формулами конечномерного случая [1].

\section{§2. Определения, вспомогательные результаты}

Пусть $X$ - локально выпуклое пространство, $\mu$ - радоновская центрированная гауссовская мера на $X, H \subset X$-пространство Камерона-Мартина меры $\mu$ с гильбертовой нормой $\|\cdot\|_{H}$.

Пусть $E$ - сепарабельное гильбертово пространство. Обозначим через $\mathscr{F} C_{b}^{\infty}(E)$ множество цилиндрических гладких функций вида

$$
F=\sum_{k=1}^{n} f_{k}\left(\left\langle x_{1}^{*}, \cdot\right\rangle, \ldots,\left\langle x_{n}^{*}, \cdot\right\rangle\right) e_{k}: X \rightarrow E
$$

где $n \in \mathbb{N}, x_{k}^{*} \in X^{*}, e_{k} \in E, f_{k} \in C_{b}^{\infty}\left(\mathbb{R}^{n}\right)$.

Пусть $D$ - производная по Фреше вдоль направлений пространства $H$ функций из $\mathscr{F} C_{b}^{\infty}(E)$.

Производные высших порядков определяются итеративно.

Введем соболевские пространства $W_{p}^{k}(E)$ как замыкание $\mathscr{F} C_{b}^{\infty}(E)$ по норме

$$
\|F\|_{p, k}=\sum_{j=1}^{k}\left\|D^{j} F\right\|_{L_{p}\left(X, \mu, H^{\otimes j} \otimes E\right)} .
$$

Замыкание оператора $D^{k}$ относительно нормы $\|\cdot\|_{p, k}$ называется стохастической производной и будет обозначаться тем же символом.

Положим

$$
\begin{gathered}
W_{\infty}^{k}(E):=\left\{f \mid \forall p \geqslant 1, \forall f \in W_{p}^{k}(E)\left\|D^{j} f\right\|_{L_{\infty}\left(X, \mu, H^{\otimes j} \otimes E\right)}<\infty, 0 \leqslant j \leqslant k\right\} \\
\|f\|_{\infty, k}:=\max _{0 \leqslant j \leqslant k}\left\|D^{k} f\right\|_{L_{\infty}\left(X, \mu, H^{\otimes k} \otimes E\right)} .
\end{gathered}
$$

Сопряженный оператор $\delta=D^{*}$, где $\mathscr{D}(D)=W_{p}^{1}(E)$,

$$
\delta=\delta_{q}: \mathscr{D}\left(\delta_{q}\right) \subset L_{q}(X, \mu, H \otimes E) \rightarrow L_{q}(X, \mu, H), \quad \frac{1}{p}+\frac{1}{q}=1
$$

называется оператором дивергенции (или расширенньм интегралом Скорохода). 
Некоторые моментные неравенства для оператора дивергенции и стохастической производной приведены в следующем утверждении.

Лемма 2.1 [7; с. 50]. Если $f \in W_{p}^{k}(H)$, то $\delta f \in W_{p}^{k-1}(\mathbb{R})$, причем существует такая константа $c_{p, k}$, что $\forall f \in W_{p}^{k}(H)$

$$
\|\delta f\|_{W_{p}^{k-1}} \leqslant c_{p, k}\|\delta f\|_{W_{p}^{k}(H)} .
$$

ОПРЕДЕЛЕНИЕ 2.1. Функция $f: X \rightarrow E$ назьвается $H$-липшичевой $c$ константой $L$, если $\forall x \in X, \forall h \in H$

$$
\|f(x+h)-f(x)\|_{E} \leqslant L\|h\|_{H} .
$$

ОПРЕДЕЛЕНИЕ 2.2. Функция $f: X \rightarrow E$ принадлежит классу $\mathscr{H} C^{n}(E)$, если для любого $x \in X$ отображение $H \ni h \rightarrow f(x+h) \in E$ является $n$ раз непрерьвно дифференцируемьм по $\Phi$ реше.

Имеет место следующий известный факт о существовании $H$-липшицевой модификации у функций из класса $W_{\infty}^{1}$ (см., например, [8]).

Лемма 2.2. Предположим, что $f \in W_{\infty}^{1}(E)$. Тогда существует H-липшицева модификация $f$ с константой $\operatorname{ess}^{\sup _{\omega}}\|D f(\omega)\|$.

Пространство Камерона-Мартина $H$ естественным образом можно отождествить с пространством квадратически интегрируемых функционалов $H(\mu)$. Обозначим через $\widehat{h}$ элемент $H(\mu)$, соответствующий вектору $h \in H$. Нам понадобится следуюшее известное утверждение о действии стохастической производной на условное математическое ожидание дифференцируемой случайной величины.

Лемма 2.3. Пусть $H_{0}$ - линейное подпространство $H, \sigma_{0}$ - $\sigma$-алгебра, порожденная функционалами $\left\{\widehat{h}, h \in H_{0}\right\}, \pi$ - ортопроектор в $H$ на $H_{0}$. Тогда для любой функции $f \in W_{p}^{k}, p \geqslant 1, k \geqslant 1$, условное математическое ожидание $\mathrm{E}\left(f / \sigma_{0}\right)$ также принадлежст $W_{p}^{k}$ и справедлива формула

$$
D \mathrm{E}\left(f / \sigma_{0}\right)=\pi \mathrm{E}\left(D f / \sigma_{0}\right) .
$$

\section{§3. Основные результаты}

Пусть $\mu$-центрированная радоновская гауссовская мера на локально выпуклом пространстве $X, H \subset X$ - пространство Камерона-Мартина, $\left\{\omega_{n}(t), t \in[0, T]\right.$, $n \geqslant 1\}$ - последовательность независимых одномерных винеровских процессов, заданных на некотором вероятностном пространстве $(\Omega, \mathscr{F}, \mathrm{P})$.

Рассмотрим следующее стохастическое дифференциальное уравнение в $X$, зависящее от начального условия $u \in X$ :

$$
\varphi_{s, t}(u)=u+\int_{s}^{t} a_{z}\left(\varphi_{s, z}(u)\right) d z+\sum_{n=1}^{\infty} \int_{s}^{t} \sigma_{z}^{n}\left(\varphi_{s, z}(u)\right) d \omega_{n}(z),
$$

г де $a:[0, T] \times X \rightarrow H, \sigma^{n}:[0, T] \times X \rightarrow H, n \geqslant 1,-$ измеримые по паре переменных функции. 
Иногда более удобно исследовать уравнение (3.1), записанное в форме Стратоновича:

$$
\varphi_{s, t}(u)=u+\int_{s}^{t} \widetilde{a}_{z}\left(\varphi_{s, z}(u)\right) d z+\sum_{n=1}^{\infty} \int_{s}^{t} \sigma_{z}^{n}\left(\varphi_{s, z}(u)\right) \circ d \omega_{n}(z)
$$

где

$$
\widetilde{a}_{t}=a_{t}-\frac{1}{2} \sum_{n}\left\langle D \sigma_{z}^{n}, \sigma_{z}^{n}\right\rangle_{H}
$$

Рассмотрим также обрашенное уравнение Стратоновича

$$
\varphi_{t, s}(u)=u-\int_{s}^{t} \widetilde{a}_{z}\left(\varphi_{z, t}(u)\right) d z+\sum_{n=1}^{\infty} \int_{s}^{t} \sigma_{z}^{n}\left(\varphi_{z, t}(u)\right) \circ d \widetilde{\omega}_{n}(z)
$$

с новьми винеровскими процессами $\widetilde{\omega}_{n}(t)=\omega_{n}(T)-\omega_{n}(t)$. Данное уравнение в конечномерном пространстве задает обратньй стохастический поток $\varphi_{t, s}, t \geqslant s[1]$.

Основным результатом настояшей статьи является следуюшая теорема.

Теорема 3.1. І. Предположим, что для всех $t \in[0, T], n \geqslant 1$ выполняется

$$
a_{t} \in W_{\infty}^{2}(H), \quad \sigma_{t}^{n} \in W_{\infty}^{3}(H)
$$

$u$

$$
\begin{aligned}
A_{j} & :=\sup _{t} \operatorname{ess} \sup \left\|D^{j} a_{t}(u)\right\|_{H^{\otimes(j+1)}}<\infty, \quad j \in\{0,1,2\}, \\
S_{l} & :=\sup _{t} \operatorname{ess} \sup \sum_{n}\left\|D^{l} \sigma_{t}^{n}(u)\right\|_{H^{\otimes(l+1)}}^{2}<\infty, \quad l \in\{0, \ldots, 3\} .
\end{aligned}
$$

Тогда существуют случайный әлемент $\varphi=\varphi_{s, t}(u, \omega):[0, T] \times[0, T] \times X \times$ $\Omega \rightarrow X$, измеримый по четверке переменных $(s, t, u, \omega)$, и множсество $\Omega_{0} \subset \Omega$, $\mathrm{P}\left(\Omega_{0}\right)=1$, такие, что для всех $\omega \in \Omega_{0}$ справедливы следуюшие утвержсдения:

а) соотношения $\left(3.1^{\prime}\right),\left(3.1^{\prime \prime}\right)$ выполняются для всех $s \leqslant t$, где стохастические интеграль рассматриваются как интеграль от $L_{2}(X, \mu, H)$ значных случайных әлементов;

b) для всех $t_{1}, t_{2}, t_{3} \in[0, T]$

$$
\begin{gathered}
\varphi_{t_{2}, t_{3}}\left(\varphi_{t_{1}, t_{2}}(u, \omega), \omega\right)=\varphi_{t_{1}, t_{3}}(u, \omega) \quad \partial \Omega \text { я } \quad \mu-n . в . \quad u \in X, \\
\varphi_{t_{1}, t_{1}}(\cdot, \omega)=\operatorname{id}_{X}
\end{gathered}
$$

c) для всех $s, t \in[0, T]$ мери $\mu_{s, t}(\omega)=\mu \circ\left(\varphi_{s, t}(\cdot, \omega)\right)^{-1}$ и $\mu$ әквивалентнь;

d) плотность Радона-Никодима $\rho_{s, t}(\omega)(u):=\frac{d \mu_{s, t}(\omega)}{d \mu}(u)$ имеет вид

$$
\rho_{t, s}(u)=\exp \left\{-\int_{s}^{t}\left(\delta \widetilde{a}_{z}\right)\left(\varphi_{s, z}(u)\right) d z-\sum_{n} \int_{s}^{t}\left(\delta \sigma_{z}^{n}\right)\left(\varphi_{s, z}(u)\right) \circ d \omega_{n}(z)\right\}
$$

$2 \partial e s \leqslant t, u$

$$
\rho_{s, t}(u)=\left(\rho_{t, s}\left(\varphi_{s, t}(u)\right)\right)^{-1} .
$$

II. Предположим, что $a_{t} \in \mathscr{H} C^{2}(H), \sigma_{t}^{n} \in \mathscr{H} C^{3}(H)$ для всех $t \in[0, T]$, $n \geqslant 1, u A_{j}, S_{k}$, определенные в первой части теоремы, конечны. Тогда существует процесс $\varphi_{s, t}$ такой, что для кажсдого $и \in X$ соотношения $\left(3.1^{\prime}\right)$, $\left(3.1^{\prime \prime}\right)$ выполняются $\mathrm{P}-n . н . ~ и \varphi_{s, t}(u)$ непрерывно по $s, t$ для $\mathrm{P}-n$. в. $\omega$. При этом любая модификация $\varphi_{s, t}(u)$, непрерывная по $s, t$, удовлетворяет условиям а)-d) первой части теоремы. 
ЗАмЕчАния. 1) Множество $\mu$-нулевой меры в b) зависит, вообше говоря, от $t_{1}$, $t_{2}, t_{3}$ и $\omega$.

2) Мы рассматриваем суперпозицию функций в (3.3), однако $\varphi_{t, s}$ определена с точностью до множеств $\mu$-нулевой меры. Данная композиция корректно определена, так как меры $\mu$ и $\mu_{t, s}$ эквивалентны по утверждению с). Суперпозиция $\varphi_{t_{2}, t_{3}} \circ \varphi_{t_{1}, t_{2}}$ в b) корректно определена по той же причине.

3) Для $\omega \in \Omega_{0}, s, t \in[0, T]$ отображение $\varphi_{s, t}(\cdot, \omega): X \rightarrow X$ является $\mu$-п.н. обратимым $\left(\right.$ см. b)) и $\varphi_{t, s}(\cdot, \omega)$ задает обратное отображение, т.е.

$$
\varphi_{t, s}\left(\varphi_{s, t}(\cdot, \omega), \omega\right)=\varphi_{s, t}\left(\varphi_{t, s}(\cdot, \omega), \omega\right)=\operatorname{id}_{X} \quad \mu \text {-п.н. }
$$

4) Стохастические интегралы Стратоновича в $\left(3.1^{\prime}\right),\left(3.1^{\prime \prime}\right),(3.2)$ рассматриваются ради простоты обозначений и означают интегралы Ито от случайных элементов со значениями в $L_{2}$ с необходимой добавкой. Например, выражение

$$
\begin{aligned}
& \int_{s}^{t}\left(\delta \sigma_{z}^{n}\right)\left(\varphi_{s, z}(u)\right) \circ d \omega_{n}(z) \text { означает } \\
& \int_{s}^{t}\left(\delta \sigma_{z}^{n}\right)\left(\varphi_{s, z}(u)\right) d \omega_{n}(z)+\frac{1}{2} \int_{s}^{t}\left\langle D\left(\delta\left(\sigma_{z}^{n}\right)\right), \sigma_{z}^{n}\right\rangle\left(\varphi_{s, z}\right) d z .
\end{aligned}
$$

\section{§4. Конечномерные аппроксимации}

Пусть $\left\{e_{m}\right\}$ - ортонормированньй базис в $H$, причем квадратически интегрируемые функционалы $\left\{\widehat{e}_{m}\right\}$ являются элементами $X^{*} \subset H(\mu)$. Линейное отображение $j: X \ni u \mapsto\left(\left\langle\widehat{e}_{1}, u\right\rangle,\left\langle\widehat{e}_{2}, u\right\rangle, \ldots\right) \in \mathbb{R}^{\infty}$ переводит меру $\mu$ в меру $\gamma-$ произведение гауссовских $\mathcal{N}(0,1)$ мер. При этом $H$ переходит в $l_{2}$, а классы $W_{p}^{k}(X, \mu, H)$ - в $W_{p}^{k}\left(\mathbb{R}^{\infty}, \gamma, l_{2}\right)$. Можно проверить существование $\gamma$-измеримого отображения $k: \mathbb{R}^{\infty} \rightarrow X$ такого, что $(k j)(u)=u \mu$-п.н. Легко видеть также, что функции $\mathbb{R}^{\infty} \ni y \mapsto j a(k y), \mathbb{R}^{\infty} \ni y \mapsto j \sigma_{n}(k y)$ удовлетворяют всем предположениям теоремы 3.1. Поэтому далее достаточно доказывать теорему в предположении $X=\mathbb{R}^{\infty}$, где $\mu=\gamma$. Пусть $\pi_{m}$ - проектор на первые $m$ координат, $\pi^{m}$ - проектор на оставшиеся координаты. Уравнение (3.1) будет аппроксимироваться следующей последовательностью:

$$
\varphi_{s, t}^{(m)}(u)=u+\int_{s}^{t} a_{z}^{m}\left(\varphi_{s, z}^{(m)}(u)\right) d z+\sum_{n} \int_{s}^{t} \sigma_{z}^{m, n}\left(\varphi_{s, z}^{(m)}(u)\right) d \omega_{n}(z),
$$

где функции $a_{z}^{m}, \sigma_{z}^{m n}$ будут такими, что

$$
a_{z}^{m}(u)=\pi_{m} a_{z}^{m}\left(\pi_{m} u\right), \quad \sigma_{z}^{m, n}=\pi_{m} \sigma_{z}^{m, n}\left(\pi_{m} u\right)
$$

Заметим, что в этом случае поток $\varphi_{s, t}^{(m)}$ изменяет лишш координаты $\pi_{m} u$ и не затрагивает $\pi^{m} u$. Поэтому если коэффициенты (4.1) удовлетворяют (4.2), то нам остается рассмотреть конечномерную задачу, детально исследованную в [1] не только для гауссовских мер, но и для мер с гладкой плотностью.

Таким образом, нашей целью является аппроксимация коэффициентов $a, \sigma^{n}$ "конечномерными" функциями $a^{m}, \sigma^{m, n}$, а затем обоснование предельного перехода для $\varphi_{s, t}^{(m)}$ и плотностей Радона-Никодима

$$
\rho_{s, t}^{(m)}:=\frac{d \mu \circ\left(\varphi_{s, t}^{m}\right)^{-1}}{d \mu}
$$


Нам понадобится следующая

Лемма 4.1 [9]. Пусть $X_{1}, X_{2}$ - два сепарабельных метрических пространства, $\mu_{i}$ - вероятностная мера на $X_{i},\left\{f_{n}: X_{1} \rightarrow X_{2}, n \geqslant 0\right\}$ - последовательность измеримых отображсний. Предположим, что

а) мерьц $\mu_{1} \circ\left(f_{n}\right)^{-1}$ абсолютно непрерывны относительно $\mu_{2}$ для кажсдого $n \geqslant 1$

b) последовательность плотностей Радона-Никодима

$$
\left\{\frac{d \mu_{1} \circ\left(f_{n}\right)^{-1}}{d \mu_{2}}, n \geqslant 1\right\}
$$

равномерно интегрируема,

c) $f_{n}$ сходится $\kappa f_{0}$ при $n \rightarrow \infty$ по мере $\mu_{1}$.

Тогда $\mu_{1} \circ\left(f_{0}\right)^{-1} \ll \mu_{2}$.

Более того, если последовательность $\left\{\frac{d \mu_{1} \circ\left(f_{n}\right)^{-1}}{d \mu_{2}}, n \geqslant 1\right\}$ сходится по мере $\mu_{2} \kappa$ некоторой функции $p$, то

$$
p=\frac{d \mu_{1} \circ\left(f_{0}\right)^{-1}}{d \mu_{2}} .
$$

Как следует из леммы 4.1, равномерная интегрируемость плотностей играет большую роль для обоснования абсолютной непрерывности предельной меры. В следующей теореме приводятся некоторые оценки для $\rho_{s, t}^{(m)}$, не зависящие от размерности пространства $m$.

ТЕОРЕМА 4.1. Пусть $\mu$ - гауссовская мера с нулевым средним и единичной матрицей ковариащий в $\mathbb{R}^{m}$. Предположим, что измеримые функиии

$$
a:[0, T] \times \mathbb{R}^{m} \rightarrow \mathbb{R}^{m}, \quad \sigma^{n}:[0, T] \times \mathbb{R}^{m} \rightarrow \mathbb{R}^{m}, \quad n \geqslant 1,
$$

удовлетворяют условиям:

(i) $\forall t \in[0, T] \quad a_{t} \in C_{b}^{2}\left(\mathbb{R}^{m}, \mathbb{R}^{m}\right), \sigma_{t}^{n} \in C_{b}^{3}\left(\mathbb{R}^{m}, \mathbb{R}^{m}\right)$;

(ii) $A_{j}:=\sup _{t, x}\left\|\nabla^{j} a_{t}(x)\right\|<\infty, j \in\{0,1,2\}$,

$$
S_{l}:=\sup _{t, x} \sum_{n}\left\|\nabla^{l} \sigma_{t}^{n}(x)\right\|^{2}<\infty, l \in\{0, \ldots, 3\} .
$$

Тогда существуют случайная функиия $\varphi=\varphi_{s, t}(u), s, t \in[0, T], u \in \mathbb{R}^{m}$, удовлетворяющая $\left(3.1^{\prime}\right),\left(3.1^{\prime \prime}\right)$ для кахсдого $и, \quad$ м мнохсество $\Omega_{0} \subset \Omega$, $\mathrm{P}\left(\Omega_{0}\right)=1$, такие, что для всех $\omega \in \Omega_{0}$ :

а) $\varphi$ непрерывна по $(s, t, u)$;

b) для всех $s, t \varphi_{s, t}(u)$ дифференцируема по $u$,

$$
\begin{aligned}
\nabla \varphi_{s, t}(u)=\operatorname{id}_{\mathbb{R}^{m}}+\int_{s}^{t} \nabla a_{z}\left(\varphi_{s, z}(u)\right) \nabla \varphi_{s, z}(u) d z \\
+\sum_{n} \int_{s}^{t} \nabla \sigma_{z}^{n}\left(\varphi_{s, z}(u)\right) \nabla \varphi_{s, z}(u) d \omega_{n}(z)
\end{aligned}
$$

для всех $s \leqslant t, u \in \mathbb{R}^{m}$

c) для всех $u \in \mathbb{R}^{m}, t_{1}, t_{2}, t_{3} \in[0, T]$

$$
\varphi_{t_{1}, t_{1}}(u, \omega)=u, \quad \varphi_{t_{2}, t_{3}}\left(\varphi_{t_{1}, t_{2}}(u, \omega), \omega\right)=\varphi_{t_{1}, t_{3}}(u, \omega)
$$


d) мерьц $\mu_{s, t}(\omega):=\mu \circ\left(\varphi_{s, t}(\cdot, \omega)\right)^{-1}$ и $\mu$ әквивалентны, причем для плотностей Радона-Никодима $\rho_{s, t}=\frac{d \mu_{s, t}}{d \mu}$ въполняются формуль $(3.2),(3.3)$, əде

$$
(\delta f)(u)=-\sum_{k=1}^{m}\left(\frac{\partial f_{k}(u)}{\partial u_{k}}-f_{k}(u) u_{k}\right)
$$

для $f=\left(f_{1}, \ldots, f_{m}\right) \in C^{1}\left(\mathbb{R}^{m}, \mathbb{R}^{m}\right), u=\left(u_{1}, \ldots, u_{m}\right) \in \mathbb{R}^{m} ;$

е) существует константа $C=C\left(A_{0}, A_{1}, S_{0}, S_{1}, S_{2}\right)$, зависящая только от $A_{j}, S_{l}$ и не зависящая от размерности пространства $\mathbb{R}^{m}$, такая, что

$$
\sup _{0 \leqslant s, t \leqslant T} \int_{\Omega} \int_{\mathbb{R}^{m}}\left|\rho_{s, t} \ln \rho_{s, t}\right| d \mu d \mathrm{P} \leqslant C
$$

ЗАмЕчАНИЕ. Если в теореме 3.1 взять $H=X=\mathbb{R}^{m}, \mu=\mathscr{N}\left(0, \mathrm{id}_{\mathbb{R}^{m}}\right)$ и коэффициенты $a, \sigma^{n}$ удовлетворяют условиям теоремы 4.1 , то все утверждения теоремы 3.1 выполняются. Действительно, в этом случае стохастическая производная является градиентом в $\mathbb{R}^{m}$, а сопряженньй оператор задается формулой (4.4).

ДокаЗАТЕЛЬство тЕОРемы 4.1. Доказательство утверждений а)-d) см., например, в [1; $\S 4.3-4.6]$. Проверим утверждение е). Рассмотрим сначала случай $s \leqslant t$. Без потери общности можно предполагать, что $s=0$. Обозначим $\varphi_{t}:=\varphi_{0, t}$.

Лемма 4.2. Пусть $(A, \mathfrak{A}, \nu)$ - вероятностное пространство. Предположим, что измеримое отображение $f: A \rightarrow A$ удовлетворяет следующим условиям:

1) мерьи $\nu$ и $f^{-1}$ әквивалентнь;

2) существует измеримое отображсение $g: A \rightarrow A$ такое, что $g(f(\alpha))=$ $f(g(\alpha))=\alpha$ для $\nu$-n.в. $\alpha \in A$.

Тогда $\nu \circ g^{-1} \sim \nu$

$$
\frac{d \nu \circ f^{-1}}{d \nu}=\left(\frac{d \nu \circ g^{-1}}{d \nu}(g)\right)^{-1}
$$

$u$

$$
\int_{A}\left|\frac{d \nu \circ f^{-1}}{d \nu} \ln \frac{d \nu \circ f^{-1}}{d \nu}\right| d \nu=\int_{A}\left|\ln \frac{d \nu \circ g^{-1}}{d \nu}\right| d \nu
$$

Обозначим через $\mathrm{E}_{\mu}, \mathrm{E}_{\mathrm{P}}, \mathrm{E}_{\mu \times \mathrm{P}}$ математические ожидания относительно мер $\mu$, $\mathrm{P}, \mu \times \mathrm{P}$ соответственно. Переходя в (3.2) от интеграла Стратоновича к интегралу 
Ито и применяя (4.7), получаем

$$
\begin{aligned}
\mathrm{E}_{\mu \times \mathrm{P}}\left|\rho_{0, t} \ln \rho_{0, t}\right|= & \mathrm{E}_{\mu \times \mathrm{P}}\left|\ln \rho_{t, 0}\right| \\
\leqslant & \mathrm{E}_{\mu \times \mathrm{P}} \int_{0}^{t}\left|\left(\delta a_{s}\right)\left(\varphi_{s}\right)\right| d s \\
& +\frac{1}{2} \mathrm{E}_{\mu \times \mathrm{P}} \int_{0}^{t}\left|\sum_{n} \delta\left(\nabla_{\sigma_{s}^{n}} \sigma_{s}^{n}\right)\left(\varphi_{s}\right)\right| d s \\
& +\mathrm{E}_{\mu \times \mathrm{P}}\left|\sum_{n} \int_{0}^{t}\left(\delta \sigma_{s}^{n}\right)\left(\varphi_{s}\right) d \omega_{n}(s)\right| \\
& +\frac{1}{2} \mathrm{E}_{\mu \times \mathrm{P}}\left|\int_{0}^{t} \sum_{n}\left(\nabla_{\sigma_{s}^{n}}\left(\delta \sigma_{s}^{n}\right)\right)\left(\varphi_{s}\right) d s\right| \\
= & I_{1}+\frac{1}{2} I_{2}+I_{3}+\frac{1}{2} I_{4} .
\end{aligned}
$$

Оценим каждое слагаемое в (4.8). Обоснование сушествования каждого из интегралов в (4.8) будет получено в процессе доказательства. Нам потребуется следующее утверждение о действии оператора дивергенции $\delta$ на композицию гладкой функции и $\varphi_{t}$.

Лемма 4.3. Предположим, что $b \in C^{1}\left(\mathbb{R}^{m}, \mathbb{R}^{m}\right)$. Тогда

$$
\begin{aligned}
(\delta b)\left(\varphi_{s}\right)=\delta( & \left.b\left(\varphi_{s}\right)\right)+\left\langle b\left(\varphi_{s}\right), \int_{0}^{s} a_{z}\left(\varphi_{z}\right) d z+\sum_{n} \int_{0}^{s} \sigma_{z}^{n}\left(\varphi_{z}\right) d \omega_{n}(z)\right\rangle \\
+ & \operatorname{tr}\left(( \nabla b ) ( \varphi _ { s } ) \left\{\int_{0}^{s}\left(\nabla a_{z}\right)\left(\varphi_{z}\right) \nabla \varphi_{z} d z\right.\right. \\
& \left.\left.+\sum_{n} \int_{0}^{s}\left(\nabla \sigma_{z}^{n}\right)\left(\varphi_{z}\right) \nabla \varphi_{z} d \omega_{n}(z)\right\}\right)
\end{aligned}
$$

ДокАЗАТЕЛьство. Заметим, что

$$
\begin{aligned}
(\delta b)\left(\varphi_{s}\right)= & \left\langle\varphi_{s}, b\left(\varphi_{s}\right)\right\rangle-\operatorname{tr}(\nabla b)\left(\varphi_{s}\right) \\
= & \left\langle u, b\left(\varphi_{s}\right)\right\rangle+\left\langle\int_{0}^{s} a_{z}\left(\varphi_{z}\right) d z+\sum_{n} \int_{0}^{s} \sigma_{z}^{n}\left(\varphi_{z}\right) d \omega_{n}(z), b\left(\varphi_{s}\right)\right\rangle \\
& -\operatorname{tr} \nabla\left(b\left(\varphi_{s}\right)\right)+\operatorname{tr}\left((\nabla b)\left(\varphi_{s}\right) \nabla \varphi_{s}-(\nabla b)\left(\varphi_{s}\right)\right) .
\end{aligned}
$$

Применяя (4.3) к (4.10), получаем выражение (4.9).

Лемма 4.3 доказана.

Применим (4.9) для оценки слагаемого $I_{1}$ в (4.8):

$$
\begin{aligned}
I_{1} \leqslant & \mathrm{E}_{\mu \times \mathrm{P}} \int_{0}^{t}\left|\delta\left(a_{s}\left(\varphi_{s}\right)\right)\right| d s+\mathrm{E}_{\mu \times \mathrm{P}} \int_{0}^{t}\left|\left\langle\int_{0}^{s} a_{z}\left(\varphi_{z}\right) d z, a_{s}\left(\varphi_{s}\right)\right\rangle\right| d s \\
& +\mathrm{E}_{\mu \times \mathrm{P}}\left|\int_{0}^{t}\left\langle a_{s}\left(\varphi_{s}\right), \sum_{n} \int_{0}^{s} \sigma_{z}^{n}\left(\varphi_{z}\right) d \omega_{n}(z)\right\rangle d s\right| \\
& +\mathrm{E}_{\mu \times \mathrm{P}}\left|\int_{0}^{t} \operatorname{tr}\left\{\nabla a_{s}\left(\varphi_{s}\right) \int_{0}^{s} \nabla a_{z}\left(\varphi_{z}\right) \nabla \varphi_{z} d z\right\} d s\right| \\
& +\mathrm{E}_{\mu \times \mathrm{P}}\left|\int_{0}^{t} \operatorname{tr}\left\{\nabla a_{s}\left(\varphi_{s}\right) \sum_{n} \int_{0}^{s} \nabla \sigma_{z}^{n}\left(\varphi_{z}\right) \nabla \varphi_{z} d \omega_{n}(z)\right\} d s\right| \\
= & I_{1,1}+\cdots+I_{1,5} .
\end{aligned}
$$


Заметим, что

$$
\begin{gathered}
I_{1,2} \leqslant \int_{0}^{t} \int_{0}^{s}\left\|a_{z}\right\|_{\infty}\left\|a_{s}\right\|_{\infty} d z d s \leqslant \frac{1}{2} A_{0}^{2} \\
\left(I_{1,3}\right)^{2} \leqslant t \int_{0}^{t}\left\|a_{s}\right\|_{\infty}^{2} \mathrm{E}_{\mu} \mathrm{E}_{\mathrm{P}}\left\|\sum_{n} \int_{0}^{s} \sigma_{z}^{n}\left(\varphi_{z}\right) d \omega_{n}(z)\right\|_{\mathbb{R}^{m}}^{2} d z d s \\
\leqslant t \int_{0}^{t}\left\|a_{s}\right\|_{\infty}^{2} \sum_{n} \int_{0}^{s}\left\|\sigma_{z}^{n}\right\|_{\infty}^{2} d z d s \leqslant t^{2} A_{0}^{2} S_{0} .
\end{gathered}
$$

Для оценки $I_{1,1}$ применим лемму 2.1:

$$
\begin{aligned}
\left(I_{1,1}\right)^{2} & \leqslant t \int_{0}^{t} \mathrm{E}_{\mathrm{P}}\left\{\mathrm{E}_{\mu}\left(\delta\left(a_{s}\left(\varphi_{s}\right)\right)\right)^{2}\right\} d s \\
& \leqslant t c_{2,1} \int_{0}^{t} \mathrm{E}_{\mathrm{P}}\left\{\mathrm{E}_{\mu}\left[\left\|a_{s}\left(\varphi_{s}\right)\right\|^{2}+\left\|\nabla a_{s}\left(\varphi_{s}\right) \nabla \varphi_{s}\right\|^{2}\right]\right\} d s \\
& \leqslant t c_{2,1} \int_{0}^{t}\left(\left\|a_{s}\right\|_{\infty}^{2}+\left\|\nabla a_{s}\right\|_{\infty}^{2} \mathrm{E}_{\mathrm{P}} \mathrm{E}_{\mu}\left\|\nabla \varphi_{s}\right\|_{\mathrm{op}}^{2}\right) d s
\end{aligned}
$$

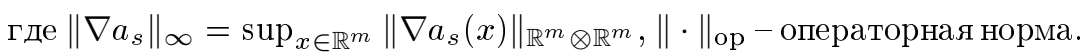

Искомое неравенство для $I_{1,1}$ вытекает из следующего утверждения.

Лемма 4.4. Для любого $p \geqslant 1$ существуют константы $K_{1}(p)=K_{1}(p, T$, $\left.A_{0}, A_{1}, S_{0}, S_{1}\right)$ u $K_{2}(p)=K_{2}\left(p, T, A_{j}, S_{j}, j=\overline{0,2}\right)$ maкие, что

$$
\mathrm{E}_{\mu \times \mathrm{P}}\left\|\nabla^{j} \varphi_{t}\right\|_{\mathrm{op}}^{p} \leqslant K_{j}(p), \quad j=1,2 .
$$

Более того, $\nabla \varphi_{t}$ обратимо п.н. и существует такое $K_{3}(p)=K_{3}\left(p, T, A_{j}, S_{j}\right.$, $j=\overline{0,2})$, что

$$
\mathrm{E}_{\mu \times \mathrm{P}}\left\|\left(\nabla \varphi_{t}\right)^{-1}\right\|_{\mathrm{op}}^{p} \leqslant K_{3}(p) .
$$

ДокАЗАТЕЛьство. Отметим, что $\left\|\nabla \varphi_{t}\right\|_{\text {op }} \leqslant 1+\left\|\nabla \varphi_{t}-\mathrm{id}\right\|_{H S}$, где $\|\cdot\|_{H S}-$ норма Гильберта-Шмидта. Положим $\psi_{t}:=\nabla \varphi_{t}-\mathrm{id}$.

Тогда

$$
\psi_{t}=\int_{0}^{t}\left(\nabla a_{s}\left(\varphi_{s}\right) \psi_{s}+\nabla a_{s}\left(\varphi_{s}\right)\right) d s+\sum_{n} \int_{0}^{t}\left(\nabla \sigma_{s}^{n}\left(\varphi_{s}\right) \psi_{s}+\nabla \sigma_{s}^{n}\left(\varphi_{s}\right)\right) d \omega_{n}(s)
$$

Применяя формулу Ито к $\left\|\psi_{t}\right\|_{H S}^{p}$ и рассуждения, аналогичные [1; с. 156], получаем следуюшее неравенство:

$$
\mathrm{E}\left\|\psi_{t}\right\|_{H S}^{p} \leqslant C\left(p, A_{1}, S_{1}\right) \int_{0}^{t}\left(1+\mathrm{E}\left\|\psi_{s}\right\|_{H S}^{p}\right) d s
$$

где $C$ не зависит от размерности пространства. Неравенство (4.12) получается применением леммы Гронуолла для $j=1$. Случай $j=2$ рассматривается аналогично. Обратимость $\nabla \varphi_{t}$ является известным фактом, $\left(\nabla \varphi_{t}\right)^{-1}$ удовлетворяет некоторому линейному стохастическому уравнению. Оценки для моментов $\left(\nabla \varphi_{t}\right)^{-1}$ получаются так же, как и для $\nabla \varphi_{t}$. Лемма 4.4 доказана. 
Для исследования $I_{1,4}, I_{1,5}$ нам понадобится следующий факт. Если $H$ - гильбертово пространство, $A, B \in \mathscr{H} \mathscr{S}(H)$ - операторы Гильберта-Шмидта и $C \in$ $\mathscr{L}(H)$ - ограниченный оператор, то

$$
|\operatorname{tr} A B| \leqslant\|A\|_{H S}\|B\|_{H S}, \quad\|A C\|_{H S} \leqslant\|A\|_{H S}\|C\|_{\text {op }} .
$$

Следовательно,

$$
\begin{aligned}
I_{1,4} & \leqslant \int_{0}^{t}\left\|\nabla a_{s}\right\|_{\infty} \int_{0}^{s}\left\|\nabla a_{z}\right\|_{\infty} \mathrm{E}_{\mu \times \mathrm{P}}\left\|\nabla \varphi_{z}\right\|_{\mathrm{op}} d z d s \leqslant \frac{1}{2} K_{1} A_{0}^{2} t^{2}, \\
I_{1,5} & \leqslant t \int_{0}^{t}\left\|\nabla a_{s}\right\|_{\infty}^{2} \mathrm{E}_{\mu} \mathrm{E}_{\mathrm{P}}\left\|\sum_{n} \int_{0}^{s} \nabla \sigma_{z}^{n}\left(\varphi_{z}\right) \nabla \varphi_{z} d \omega_{n}(z)\right\|_{H S}^{2} d s \\
& \leqslant t \int_{0}^{t} A_{1}^{2} \mathrm{E}_{\mu} \mathrm{E}_{\mathrm{P}} \int_{0}^{s} \sum_{n}\left\|\nabla \sigma_{z}^{n}\left(\varphi_{z}\right) \nabla \varphi_{z}\right\|_{H S}^{2} d z d s \\
& \leqslant A_{1}^{2} t \int_{0}^{t} \int_{0}^{s} \sum_{n}\left\|\nabla \sigma_{z}^{n}\right\|_{\infty}^{2} \mathrm{E}_{\mu \times \mathrm{P}}\left\|\nabla \varphi_{z}\right\|_{\mathrm{op}}^{2} d z d s \leqslant \frac{1}{2} A_{1}^{2} t^{3} S_{0} K_{2} .
\end{aligned}
$$

Таким образом, данное неравенство завершает доказательство оценки

$$
I_{1} \leqslant K\left(T, A_{0}, A_{1}, S_{0}, S_{1}\right) .
$$

Аналогично предыдущим рассуждениям можно показать, что $I_{2} \leqslant K\left(T, A_{0}, A_{1}\right.$, $\left.S_{0}, S_{1}, S_{2}\right)$. Для этого достаточно заметить, что для каждого $t \in[0, T]$ функция $\sum_{n} \nabla_{\sigma_{t}^{n}} \sigma_{t}^{n}$ непрерывно дифференцируема и

$$
\sup _{t}\left(\left\|\sum_{n} \nabla_{\sigma_{t}^{n}} \sigma_{t}^{n}\right\|_{\mathbb{R}^{m}}+\left\|\nabla \sum_{n}\left(\nabla_{\sigma_{t}^{n}} \sigma_{t}^{n}\right)\right\|_{\mathbb{R}^{m} \otimes \mathbb{R}^{m}}\right)<\infty .
$$

Действительно,

$$
\begin{gathered}
\left\|\sum_{n} \nabla_{\sigma_{t}^{n}} \sigma_{t}^{n}\right\| \leqslant \sum_{n}\left\|\nabla \sigma_{t}^{n}\right\|\left\|\sigma_{t}^{n}\right\| \leqslant \sum_{n}\left(\left\|\nabla \sigma_{t}^{n}\right\|^{2}+\left\|\sigma_{t}^{n}\right\|^{2}\right) \leqslant S_{0}+S_{1}, \\
\left\|\nabla\left(\sum_{n} \nabla_{\sigma_{t}^{n}} \sigma_{t}^{n}\right)\right\| \leqslant \sum_{n}\left(\left\|\nabla^{2} \sigma_{t}^{n}\right\|\left\|\sigma_{t}^{n}\right\|+\left\|\nabla \sigma_{t}^{n}\right\|^{2}\right) \leqslant S_{0}+S_{1}+S_{2} .
\end{gathered}
$$

Рассмотрим $I_{3}$ :

$$
\begin{aligned}
\left|I_{3}\right| \leqslant & \mathrm{E}_{\mu \times \mathrm{P}}\left|\sum_{n} \int_{0}^{t} \delta\left(\sigma_{s}^{n}\left(\varphi_{s}\right)\right) d \omega_{n}(s)\right| \\
& +\mathrm{E}_{\mu \times \mathrm{P}}\left|\sum_{n} \int_{0}^{t}\left\langle\sigma_{s}^{n}\left(\varphi_{s}\right), \int_{0}^{s} a_{z}\left(\varphi_{z}\right) d z\right\rangle d \omega_{n}(s)\right| \\
& +\mathrm{E}_{\mu \times \mathrm{P}}\left|\sum_{n} \int_{0}^{t}\left\langle\sigma_{s}^{n}\left(\varphi_{s}\right), \sum_{m} \int_{0}^{s} \sigma_{z}^{m}\left(\varphi_{z}\right) d \omega_{m}(z)\right\rangle d \omega_{n}(s)\right| \\
& +\mathrm{E}_{\mu \times \mathrm{P}}\left|\operatorname{tr}\left\{\sum_{n} \int_{0}^{t} \nabla \sigma_{s}^{n}\left(\varphi_{s}\right) \int_{0}^{s} \nabla a_{z}\left(\varphi_{z}\right) \nabla \varphi_{z} d z d \omega_{n}(s)\right\}\right| \\
& +\mathrm{E}_{\mu \times \mathrm{P}}\left|\operatorname{tr}\left\{\sum_{n} \int_{0}^{t} \nabla \sigma_{s}^{n}\left(\varphi_{s}\right) \int_{0}^{s} \sum_{m} \nabla \sigma_{z}^{m}\left(\varphi_{z}\right) \nabla \varphi_{z} d \omega_{m}(z) d \omega_{n}(s)\right\}\right| \\
= & I_{3,1}+\cdots+I_{3,5} ;
\end{aligned}
$$




$$
\begin{aligned}
\left(I_{3,1}\right)^{2} & \leqslant \mathrm{E}_{\mu}\left\{\mathrm{E}_{\mathrm{P}}\left(\sum_{n} \int_{0}^{t} \delta\left(\sigma_{s}^{n}\left(\varphi_{s}\right)\right) d \omega_{n}(s)\right)^{2}\right\} \\
& =\mathrm{E}_{\mu \times \mathrm{P}} \sum_{n} \int_{0}^{t}\left(\delta\left(\sigma_{s}^{n}\left(\varphi_{s}\right)\right)\right)^{2} d s
\end{aligned}
$$

Дальнейшие рассуждения для $I_{3,1}$ проводятся так же, как и для $I_{1,1}$ :

$$
\begin{aligned}
\left(I_{3,2}\right)^{2} & \leqslant \mathrm{E}_{\mu \times \mathrm{P}} \sum_{n} \int_{0}^{t}\left(\left\langle\sigma_{s}^{n}\left(\varphi_{s}\right), \int_{0}^{s} a_{z}\left(\varphi_{z}\right) d z\right\rangle\right)^{2} d s \\
& \leqslant \sum_{n} \int_{0}^{t}\left\|\sigma_{s}^{n}\right\|_{\infty}^{2} d s\left(\int_{0}^{t}\left\|a_{z}\right\|_{\infty} d z\right)^{2} \leqslant S_{0} A_{0}^{2} t^{3} ; \\
\left(I_{3,3}\right)^{2} & \leqslant \mathrm{E}_{\mu} \mathrm{E}_{\mathrm{P}} \sum_{n} \int_{0}^{t}\left(\left\langle\sigma_{s}^{n}\left(\varphi_{s}\right), \sum_{m} \int_{0}^{s} \sigma_{z}^{m}\left(\varphi_{z}\right) d \omega_{m}(z)\right\rangle\right) d s \\
& \leqslant \int_{0}^{t} \sum_{n}\left\|\sigma_{s}^{n}\right\|_{\infty}^{2} \mathrm{E}_{\mu}\left(\mathrm{E}_{\mathrm{P}}\left\|\sum_{m} \int_{0}^{s} \sigma_{z}^{m}\left(\varphi_{z}\right) d \omega_{m}(z)\right\|^{2}\right) d s \leqslant \frac{1}{2} S_{0}^{2} t^{2} .
\end{aligned}
$$

Применяя (4.13) и лемму 4.4 к $I_{3,4}$, получаем

$$
\begin{aligned}
\left(I_{3,4}\right)^{2} & \leqslant \mathrm{E}_{\mu} \mathrm{E}_{\mathrm{P}} \sum_{n} \int_{0}^{t}\left(\operatorname{tr}\left\{\nabla \sigma_{s}^{n}\left(\varphi_{s}\right) \int_{0}^{s} \nabla a_{z}\left(\varphi_{z}\right) \nabla \varphi_{z} d z\right\}\right)^{2} d s \\
& \leqslant \sum_{n} \int_{0}^{t}\left\|\nabla \sigma_{s}^{n}\right\|_{\infty}^{2} s \int_{0}^{s}\left\|\nabla a_{z}\right\|_{\infty}^{2} \mathrm{E}_{\mu \times \mathrm{P}}\left\|\nabla \varphi_{z}\right\|_{\mathrm{op}}^{2} d z d s \leqslant \frac{1}{3} K_{1}(2) S_{1} A_{1}^{2} t^{3}
\end{aligned}
$$

где константа $K_{1}(2)$ определена в лемме 4.4 .

Аналогично,

$$
\left(I_{3,5}\right)^{2} \leqslant \sum_{n} \int_{0}^{t}\left\|\nabla \sigma_{s}^{n}\right\|_{\infty}^{2} \int_{0}^{s} \sum_{m}\left\|\nabla \sigma_{z}^{m}\right\|_{\infty}^{2} \mathrm{E}_{\mu \times \mathrm{P}}\left\|\nabla \varphi_{z}\right\|_{\mathrm{op}}^{2} d z d s \leqslant \frac{1}{2} S_{1}^{2} t^{2} \cdot K_{1}(2)
$$

Оценим последнее слагаемое в (4.8):

$$
\begin{aligned}
I_{4} & =\mathrm{E}_{\mu \times \mathrm{P}} \int_{0}^{t}\left|\sum_{n}\left\langle\nabla\left(\left(\delta \sigma_{s}^{n}\right)\left(\varphi_{s}\right)\right),\left(\nabla \varphi_{s}\right)^{-1} \sigma_{s}^{n}\left(\varphi_{s}\right)\right\rangle\right| d s \\
& \leqslant \int_{0}^{t} \sum_{n} \mathrm{E}_{\mu \times \mathrm{P}}\left\|\nabla\left(\left(\delta \sigma_{s}^{n}\right)\left(\varphi_{s}\right)\right)\right\|^{2} d s+\int_{0}^{t} \sum_{n}\left\|\sigma_{s}^{n}\right\|_{\infty}^{2} \mathrm{E}_{\mu \times \mathrm{P}}\left\|\left(\nabla \varphi_{s}\right)^{-1}\right\|_{\mathrm{op}}^{2} d s .
\end{aligned}
$$

Второе слагаемое ограничено по лемме 4.4. Рассмотрим подынтегральное выра- 
жение в первом члене. По лемме 4.3 имеем

$$
\begin{aligned}
\sum_{n} \mathrm{E}_{\mu \times \mathrm{P}}\left\|\nabla\left(\left(\delta \sigma_{n}^{s}\right)\left(\varphi_{s}\right)\right)\right\|^{2} \\
\leqslant 5 \sum_{n} \mathrm{E}_{\mu \times \mathrm{P}}\left(\left\|(\nabla \delta)\left(\sigma_{s}^{n}\left(\varphi_{s}\right)\right)\right\|^{2}+\left\|\nabla\left(\left\langle\sigma_{s}^{n}\left(\varphi_{s}\right), \int_{0}^{s} a_{z}\left(\varphi_{z}\right) d z\right\rangle\right)\right\|^{2}\right. \\
+\left\|\nabla\left(\left\langle\sigma_{s}^{n}\left(\varphi_{s}\right), \int_{0}^{s} \sum_{m} \sigma_{z}^{m}\left(\varphi_{z}\right) d \omega_{m}(z)\right\rangle\right)\right\|^{2} \\
+\left\|\nabla\left(\operatorname{tr}\left\{\left\langle\sigma_{s}^{n}\left(\varphi_{s}\right), \int_{0}^{s} a_{z}\left(\varphi_{z}\right) d z\right\rangle\right\}\right)\right\|^{2} \\
\left.+\left\|\nabla\left(\operatorname{tr}\left\{\left\langle\sigma_{s}^{n}\left(\varphi_{s}\right), \sum_{m} \int_{0}^{s} \sigma_{z}^{m}\left(\varphi_{z}\right) d \omega_{m}(z)\right\rangle\right\}\right)\right\|^{2}\right) \\
=5\left(I_{4,1}+\cdots+I_{4,5}\right) .
\end{aligned}
$$

Применяя леммы 4.4 и 2.1 к $I_{4,1}$, получаем неравенство $I_{4,1} \leqslant C\left(T, A_{j}, S_{j}, j=\right.$ $0,1,2)$. Ограниченность $I_{4,2}$ получается с помощью таких же рассуждений, как и для $I_{1,3}$. Можно показать $[1 ; \S 4.6]$, что

$$
\nabla\left(\sum_{m} \int_{0}^{s} \sigma_{z}^{m}\left(\varphi_{z}\right) d \omega_{m}(z)\right)=\sum_{m} \int_{0}^{s} \nabla \sigma_{z}^{m}\left(\varphi_{z}\right) \nabla \varphi_{z} d \omega_{m}(z)
$$

Следовательно,

$$
\begin{aligned}
I_{4,3} \leqslant & \sum_{n} \mathrm{E}_{\mu \times \mathrm{P}}\left\|\left\langle\nabla \sigma_{s}^{n}\left(\varphi_{s}\right) \nabla \varphi_{s}, \sum_{m} \int_{0}^{s} \sigma_{z}^{m}\left(\varphi_{z}\right) d \omega_{m}(z)\right\rangle\right\|^{2} \\
& +\sum_{n} \mathrm{E}_{\mu \times \mathrm{P}}\left\|\left\langle\sigma_{s}^{n}\left(\varphi_{s}\right), \sum_{m} \int_{0}^{s} \nabla \sigma_{z}^{m}\left(\varphi_{z}\right) \nabla \varphi_{z} d \omega_{m}(z)\right\rangle\right\|^{2} \\
\leqslant & S_{1} \mathrm{E}_{\mu \times \mathrm{P}}\left\|\nabla \varphi_{s}\right\|_{\mathrm{op}}^{2}\left\|\sum_{m} \int_{0}^{s} \sigma_{z}^{m}\left(\varphi_{z}\right) d \omega_{m}(z)\right\|^{2} \\
& +S_{0} \mathrm{E}_{\mu \times \mathrm{P}} \int_{0}^{s} \sum_{m}\left\|\nabla \sigma_{z}^{m}\left(\varphi_{z}\right)\right\|_{\mathbb{R}^{m} \otimes \mathbb{R}^{m}}^{2}\left\|\nabla \varphi_{z}\right\|_{\mathrm{op}}^{2} d z \\
\leqslant & S_{1}\left(\mathrm{E}_{\mu \times \mathrm{P}}\left\|\nabla \varphi_{s}\right\|_{\mathrm{op}}^{4}\right)^{1 / 2}\left(\mathrm{E}_{\mu \times \mathrm{P}}\left\|\sum_{m} \int_{0}^{s} \sigma_{z}^{m}\left(\varphi_{z}\right) d \omega_{m}(z)\right\|^{4}\right)^{1 / 2} \\
& +S_{0} S_{1} s K_{1}(2) .
\end{aligned}
$$

Нам понадобится следующее известное мартингальное неравенство:

$$
\mathrm{E}\left\|M_{t}\right\|^{4} \leqslant K \mathrm{E}\left(\left\langle M_{t}, M_{t}\right\rangle\right)^{2}
$$

где $M_{t}$ - непрерьвньй квадратически интегрируемьй мартингал, принимающий значения в гильбертовом пространстве и имеющий нулевое среднее. Здесь $K-$ некоторая универсальная константа.

Следовательно,

$$
\mathrm{E}_{\mu \times \mathrm{P}}\left\|\sum_{m} \int_{0}^{s} \sigma_{z}^{m}\left(\varphi_{z}\right) d \omega_{m}(z)\right\|^{4} \leqslant K\left(\sum_{m} \int_{0}^{s}\left\|\sigma_{z}^{m}\right\|_{\infty}^{2} d z\right)^{2} \leqslant K s^{2} S_{0}^{2}
$$


Учитывая все предыдущие неравенства, мы получаем ограниченность $I_{4,3}$. Выражения $I_{4,4}$ и $I_{4,5}$ рассматриваются аналогично $I_{4,3}$ с применением неравенства (4.13).

Теорема 4.1 полностью доказана.

\section{§5. Существование измеримого решения}

Предположим, что для любого $t \in[0, T], n \geqslant 1$,

а) функции $a_{t}, \sigma_{t}^{n}: X \rightarrow H$ являются $H$-липшицевьпи и

b) $\sup _{t \in[0, T]} \sup _{x \in X} \sup _{h \in H, h \neq 0}\left(\left\|a_{t}(x)\right\|+\sum_{n}\left\|\sigma_{t}^{n}(x)\right\|^{2}\right.$

$$
\left.+\frac{\left\|a_{t}(x+h)-a_{t}(x)\right\|}{\|h\|}+\sum_{n} \frac{\left\|\sigma_{t}^{n}(x+h)-\sigma_{t}^{n}(x)\right\|^{2}}{\|h\|^{2}}\right)<\infty .
$$

В этом параграфе мы докажем существование случайного элемента

$$
\varphi=\varphi_{s, t}(u, \omega), \quad 0 \leqslant s \leqslant t \leqslant T, \quad u \in X, \quad \omega \in \Omega
$$

такого, что

1) $\varphi$ измеримо по четверке аргументов $(s, t, u, \omega)$,

2) для каждого $u \in X, 0 \leqslant s \leqslant t \leqslant T$, выполняется соотношение (3.1),

3) для каждого $u \in X, \omega \in \Omega$, функция $\varphi_{s, t}(u)$ непрерывна по $s, t$.

Отметим, что если функции $a_{t}, \sigma_{t}^{n}$ удовлетворяют условиям теоремы 3.1.II, то они удовлетворяют условиям a), b).

Заметим, что для любого фиксированного $u \in X$ решение (3.1) существует. Действительно, в этом случае $\varphi_{s, t}(u)=u+\psi_{s, t}(u)$, где $\psi_{s, t}$ - решение следуюшего стохастического дифференциального уравнения в $H$ с $H$-липшицевыми коэффициентами:

$$
\psi_{s, t}(u)=\int_{s}^{t} a_{z}\left(\psi_{s, z}(u)+u\right) d z+\sum_{n} \int_{s}^{t} \sigma_{z}^{n}\left(\psi_{s, z}(u)+u\right) d \omega_{n}(z) .
$$

Применяя теорему Колмогорова, легко проверить сушествование непрерывной по $s, t$ модификации. Однако полученный процесс не обязан быть измеримым по $(s, t, u, \omega)$. Для построения процесса, измеримого по четверке аргументов, нам потребуется следующая теорема.

ТЕОРема $5.1[10]$. Пусть $(\Omega, \mathscr{F}, \mathrm{P})$ - вероятностное пространство, $Y$ - сепарабельное метрическое пространство, $(A, \mathfrak{A})$ - измеримое пространство. Рассмотрим последовательность $\mathscr{F} \otimes \mathfrak{A - и з м е р и м ы х ~ п р о ч е с с о в ~} X_{n}=X_{n}(a)$, $a \in A, n \geqslant 1$, принимающих значения в $Y$. Предположим, что для кажсдого $a \in A$ существует предел по вероятности $\left\{X_{n}(a), n \geqslant 1\right\}$.

Тогда существует $\mathscr{F} \otimes \mathfrak{A - и з м е р и м ы и ̆ ~ с л у ч а и ̆ н ы и ̆ ~ ә л е м е н т ~} X(a), a \in A$, принимающий значения в $Y$, такой, что

$$
\forall a \in A \quad X_{n}(a) \stackrel{\mathrm{P}}{\longrightarrow} X(a), \quad n \rightarrow \infty
$$


Пусть $s \in[0, T]$ фиксировано. Построим сначала случайный процесс $\varphi_{(s), t}(u, \omega)$, $t \in[s, T]$, измеримый по $(t, u, \omega)$, удовлетворяющий $(3.1)$ и непрерывный по $t$ для всех $u, \omega$.

Без потери общности считаем, что $s=0$. Пусть $\varphi_{t}-$ решение $(3.1)$ с $s=0$. Измеримая версия $\varphi_{t}$ будет построена при помощи предельного перехода с использованием теоремы 5.1.

Положим

$\varphi_{t}^{(0)}(u, \omega):=u, \quad \varphi_{t}^{(k+1)}(u, \omega):=u+\int_{0}^{t} a_{z}\left(\varphi_{z}^{(k)}(u)\right) d z+\sum_{n} \int_{0}^{t} \sigma_{z}^{n}\left(\varphi_{z}^{(k)}(u)\right) d \omega_{n}(z)$.

Возьмем в качестве $A$ пространство $C([0, T], H)$. Проверим существование $\mathscr{F} \otimes \mathscr{B}(X)$-измеримой версии интеграла $\int_{0}^{t} \sigma_{z}^{n}\left(\varphi_{z}^{(k)}(u)\right) d \omega_{n}(z)$, рассматриваемого как случайный элемент со значениями в $C([0, T], H)$.

Определим следующую последовательность $C([0, T], H)$-значных $\mathscr{F} \otimes \mathscr{B}(X)$ измеримых случайных элементов:

$$
\begin{aligned}
\xi_{t}^{k, m, n}(u)= & \sum_{j=0}^{l-1} \sigma_{j T / m}^{(n)}\left(\varphi_{j T / m}^{(k)}(u)\right)\left(\omega_{n}\left(\frac{(j+1) T}{m}\right)-\omega_{n}\left(\frac{j T}{m}\right)\right) \\
& +\sigma_{l T / m}^{(n)}\left(\varphi_{l T / m}^{(n)}(u)\right)\left(\omega_{n}(t)-\omega_{n}\left(\frac{l T}{m}\right)\right),
\end{aligned}
$$

где $l$ такое, что $t \in[l T / m,(l+1) T / m]$.

Легко проверить, что $\xi^{k, m, n}(u)$ сходится в $C([0, T], H)$ для каждого $u \in X$ по мере Р. Поэтому по теореме 5.1 существует измеримая по $(u, \omega)$ версия интеграла $\int_{0}^{\cdot} \sigma_{z}^{(n)}\left(\varphi_{z}^{(k)}(u)\right) d \omega_{n}(z)$, рассматриваемая как случайный элемент в $C([0, T], H)$. Аналогичным образом можно показать сушествование измеримой версии суммы

$$
\sum_{n} \int_{0}^{t} \sigma_{z}^{n}\left(\varphi_{z}^{(k)}(u)\right) d \omega_{n}(z)
$$

Для каждого $u \in X$ последовательность итераций $\left(\varphi_{t}^{(k)}(u)-u\right)$ сходится к $\left(\varphi_{t}(u)-u\right)$ в пространстве $C([0, T], H)$ по мере $\mathrm{P}$.

Применяя теорему 5.1 еще раз, получаем существование измеримой модификации $\varphi(s), t(u)$ для каждого $s \in[0, T]$.

Рассмотрим $\mathscr{B}(X) \otimes \mathscr{F}$-измеримый $C(\{(s, t), 0 \leqslant s \leqslant t \leqslant T\}, H)$-значный случайный элемент

$$
\varphi_{s, t}^{(n)}= \begin{cases}\varphi_{(0), t}, & \text { если } s \in[0, T / n], \\ \varphi_{((k-1) T / n), t}, & \text { если } s=k T / n, \\ \varphi_{((k-1) T / n), t} \theta_{n}+\varphi_{(k T / n), t}\left(1-\theta_{n}\right), & \text { если } s \in(k T / n,(k+1) T / n),\end{cases}
$$

где $\theta_{n}=(s-k T / n) n$.

Для каждого $u \in X$ существует непрерывная по $(s, t), 0 \leqslant s \leqslant t \leqslant T$, версия $\varphi_{s, t}(u)$. Следовательно, для любого $u \in X$ имеем

$$
\sup _{0 \leqslant s \leqslant t \leqslant T}\left\|\varphi_{s, t}^{(n)}(u, \omega)-\varphi_{s, t}(u, \omega)\right\|_{H} \rightarrow 0, \quad n \rightarrow \infty, \quad \text { для п.в. } \omega .
$$

По теореме 5.1 сушествует модификация $\varphi_{s, t}$, непрерывная по $s, t$ и измеримая по $u, \omega$. Процесс, имеюший непрерывные траектории, измерим, значит, отображение $(s, t, u, \omega) \mapsto \varphi_{s, t}(u, \omega), s \leqslant t, u \in X, \omega \in \Omega$, измеримо. 
ЗАмЕчАнИЕ. Подобно приведенным выше рассуждениям можно показать существование процесса $\varphi_{s, t}, 0 \leqslant s, t \leqslant T$, измеримого по $(s, t, u, \omega)$ и удовлетворяюшего $\left(3.1^{\prime}\right),\left(3.1^{\prime \prime}\right)$ для всех $s \leqslant t, u \in X$.

\section{§6. Доказательство теоремы 3.1}

Пусть $\widehat{a}, \widehat{\sigma}^{n}-H$-липшицевы модификации $a, \sigma^{n}$ соответственно (лемма 2.2). Рассмотрим стохастическое уравнение

$$
\varphi_{s, t}(u)=u+\int_{s}^{t} \widehat{a}_{z}\left(\varphi_{s, z}(u)\right) d z+\sum_{n} \int_{s}^{t} \widehat{\sigma}_{z}^{n}\left(\varphi_{s, z}(u)\right) d \omega_{n}(z) .
$$

Пусть $\varphi_{s, t}$ удовлетворяет условиям измеримости, полученным в $\oint 5$. Заметим, что если $\mu \circ \varphi_{s, t}^{-1} \sim \mu$, то $\varphi_{s, t}$ является также решением $(3.1)$, кроме того, $\varphi_{s, t}(u)$ будет удовлетворять (3.1) для $\mu$-п.в. $u \in X$. Поэтому далее везде предполагается, что $a=\widehat{a}, \sigma^{n}=\widehat{\sigma}^{n}$. Мы покажем, что $\varphi_{s, t}(u)$ - искомый процесс.

Так же как и в $\S 4$, предполагаем, что $X=\mathbb{R}^{\infty}, \mu$ - произведение стандартных гауссовских мер, $H=l_{2}$. Пусть $\pi_{m}$-проектор на пространство, порожденное первьми $m$ координатами, $\pi^{m}$ - проектор на оставшиеся координаты $\left(\pi_{m}, \pi^{m}\right.$ будут рассматриваться как операторы в $\mathbb{R}^{\infty}$ или в $\left.l_{2}\right)$. Отождествим $\pi_{m}\left(\mathbb{R}^{\infty}\right)$ с $\mathbb{R}^{m}$ и каждый элемент $u \in \mathbb{R}^{\infty}$ с парой $\left(u_{m}, u^{m}\right)=\left(\pi_{m}(u), \pi^{m}(u)\right) \in \mathbb{R}^{m} \times \pi^{m}\left(\mathbb{R}^{\infty}\right)$. Обозначим $\sigma$-алгебру, порожденную первыми $m$ координатными функциями, чеpeз $\mathscr{F}_{m}$.

Рассмотрим функции

$$
a^{m}=\mathrm{E}_{\mu}\left(\pi_{m} a / \mathscr{F}_{m}\right), \quad \sigma^{m, n}=\mathrm{E}_{\mu}\left(\pi_{m} \sigma^{n} / \mathscr{F}_{m}\right),
$$

где $\mathrm{E}_{\mu}\left(\cdot / \mathscr{F}_{m}\right)$ - условное математическое ожидание в $(X, \mathscr{B}(X), \mu)$ относительHO $\mathscr{F}_{m}$.

Из леммы 2.3 следует, что $a^{m}, \sigma^{m, n}, n \geqslant 1$, удовлетворяют условиям теоремы 3.1 с теми же константами. Более того, для каждого $t \in[0, T]$ имеет место сходимость

$$
\begin{aligned}
D^{i} a_{t}^{m} & \rightarrow D^{i} a_{t}, \quad m \rightarrow \infty, \quad i=0,1, \\
D^{j} \sigma_{t}^{m, n} & \rightarrow D^{j} \sigma_{t}^{n}, \quad m \rightarrow \infty, \quad j=0,1,2,
\end{aligned}
$$

для $\mu$-п.в. $u \in X$.

Заметим, что функции $a_{t}^{m}, \sigma_{t}^{m, n}$ принимают значения в $\mathbb{R}^{m}$ и зависят только от координаты $\mathbb{R}^{m}$, т.е. $\forall v_{1}, v_{2} \in \pi^{m}\left(\mathbb{R}^{\infty}\right), u_{m} \in \mathbb{R}^{m}, t \in[0, T]$

$$
a_{t}^{m}\left(u_{m}, v_{1}\right)=a_{t}^{m}\left(u_{m}, v_{2}\right), \quad \sigma_{t}^{m, n}\left(u_{m}, v_{1}\right)=\sigma_{t}^{m, n}\left(u_{m}, v_{2}\right) .
$$

Поэтому иногда, для удобства, $a^{m}, \sigma^{m, n}$ будут рассматриваться как функции, действующие из $[0, T] \times \mathbb{R}^{m}$ в $\mathbb{R}^{m}$.

Мы еше не можем применять теорему 4.1 к уравнению

$$
\begin{gathered}
d \varphi_{s, t}^{m}=a_{t}^{m}\left(\varphi_{s, t}^{m}\right) d t+\sum_{n} \sigma_{t}^{m, n}\left(\varphi_{s, t}^{m}\right) d \omega_{n}(t) \\
\varphi_{s, s}^{m}\left(u_{m}\right)=u_{m}, \quad u_{m} \in \mathbb{R}^{m}
\end{gathered}
$$


так как для каждого $t \in[0, T]$ функции $a_{t}^{m}, \sigma_{t}^{m, n}$, рассматриваемые как функции на $\mathbb{R}^{m}$, не принадлежат пространству $C_{b}^{2}$, а только $W_{\infty}^{2}$.

Известно, что элемент $f \in W_{\infty}^{k}\left(\mathbb{R}^{m}, \mu_{m}, \mathbb{R}^{m}\right), \mu_{m} \sim \mathcal{N}\left(0, \mathrm{id}_{\mathbb{R}^{m}}\right)$, имеет модификацию, которая $k-1$ раз непрерывно дифференцируема и $\nabla^{k-1} f$ глобально липшицева с константой, не превышающей $\|f\|_{\infty, k}$.

Аппроксимируем $a_{t}^{m}, \sigma_{t}^{m, n}$ дважды непрерьвно дифференцируемыми функциями. Нам понадобится следуюшее известное утверждение о приближении функции свертками.

Лемма 6.1. Пусть $\varphi: \mathbb{R}^{m} \rightarrow \mathbb{R}$ - неотрицательная бесконечно дифференцируемая функиия, $\int_{\mathbb{R}^{m}} \varphi(x) d x=1$. Положим $\varphi_{n}(x)=n^{m} \varphi(x / n), x \in \mathbb{R}^{m}$, $n \geqslant 1$.

Тогда для любой ограниченной измеримой функиии $f: \mathbb{R}^{m} \rightarrow \mathbb{R}^{m}$ имеет место сходимость

$$
f * \varphi_{n} \rightarrow f, \quad n \rightarrow \infty, \quad \text { nо мере } \mu_{m}
$$

Если $f$ равномерно непрерывна, то $f * \varphi_{n}$ сходится $\kappa f$ равномерно.

Ecлu $f \in W_{\infty}^{k}\left(\mathbb{R}^{m}, \mu_{m}, \mathbb{R}^{m}\right)$, mo

$$
\nabla^{j}\left(f * \varphi_{n}\right) \rightarrow \nabla^{j} f, \quad n \rightarrow \infty, \quad \text { для } \quad j=0, \ldots, k-1
$$

равномерно на $\mathbb{R}^{m}$,

$$
\nabla^{k}\left(f * \varphi_{n}\right) \stackrel{\mu_{m}}{\longrightarrow} \nabla^{k} f, \quad n \rightarrow \infty
$$

ЗАмЕчАниЕ. Если $f \in W_{\infty}^{k}$, то для каждого $j \in\{0, \ldots, k\}$ и $n \geqslant 1$

$$
\left\|\nabla^{j}\left(f * \varphi_{n}\right)\right\|_{\infty} \leqslant\left\|\nabla^{j} f\right\|_{\infty}
$$

Применяя диагональный метод Кантора, теорему Лебега об ограниченной сходимости и лемму 6.1, выберем подпоследовательность $\left\{k_{m}\right\}$ так, чтобы функции $a^{m, k_{m}}=a^{m} * \varphi_{k_{m}}, \sigma^{n, m, k_{m}}=\sigma^{n, m} * \varphi_{k_{m}}$ удовлетворяли условиям

$$
\begin{aligned}
\int_{0}^{T}\left\|a_{t}^{m, k_{m}}-a_{t}\right\|_{H} d t \rightarrow 0, \quad m \rightarrow \infty, \quad \mu \text {-п.н. } \\
\int_{0}^{T}\left\|\sigma_{t}^{n, m, k_{m}}-\sigma_{t}^{n}\right\|_{H}^{2} d t \rightarrow 0, \quad m \rightarrow \infty, \quad \mu \text {-п.н. }
\end{aligned}
$$

ЗАмечАниЕ. Сейчас $a^{m, k_{m}}, \sigma^{n, m, k_{m}}$ рассматриваются как функции на $X$.

Далее будут рассматриваться только функции $a^{m, k_{m}}, \sigma^{n, m, k_{m}}$, а не $a^{m}, \sigma^{n, m}$. Поэтому для удобства индекс $k_{m}$ будет опускаться.

Проверим, что для всех $s, t, 0 \leqslant s \leqslant t \leqslant T$,

$$
\mathrm{E}_{\mu \times \mathrm{P}}\left\|\varphi_{s, t}^{m}-\varphi_{s, t}\right\|_{H}^{2} \rightarrow 0, \quad m \rightarrow \infty
$$

где $\varphi_{s, t}^{m}-$ решение $(6.2)$ с

$$
\varphi_{s, s}^{m}(u)=u, \quad a_{t}^{m}(u)=\left(a_{t}^{m}\left(u_{m}\right), 0\right), \quad \sigma^{m, n}(u)=\left(\sigma^{m, n}\left(u_{m}\right), 0\right) .
$$


Действительно,

$$
\begin{aligned}
\mathrm{E}_{\mu \times \mathrm{P}}\left\|\varphi_{s, t}^{m}-\varphi_{s, t}\right\|_{H}^{2} \leqslant & 2 \int_{s}^{t} \mathrm{E}_{\mu \times \mathrm{P}}\left\|a^{m}\left(\varphi_{s, z}^{m}\right)-a\left(\varphi_{s, z}\right)\right\|_{H}^{2} d z \\
& +2 \int_{s}^{t} \sum_{n} \mathrm{E}_{\mu \times \mathrm{P}}\left\|\sigma^{m, n}\left(\varphi_{s, z}^{m}\right)-\sigma^{n}\left(\varphi_{s, z}\right)\right\|_{H}^{2} d z
\end{aligned}
$$

Первый член в (6.6) не превосходит

$$
\begin{aligned}
& 2 \int_{s}^{t} \mathrm{E}_{\mu \times \mathrm{P}}\left\|a_{z}^{m}\left(\varphi_{s, z}^{m}\right)-a_{z}\left(\varphi_{s, z}^{m}\right)\right\|_{H}^{2} d z+2 \int_{s}^{t} \mathrm{E}_{\mu \times \mathrm{P}}\left\|a_{z}\left(\varphi_{s, z}^{m}\right)-a_{z}\left(\varphi_{s, z}\right)\right\|_{H}^{2} d z \\
& \quad \leqslant 2 \int_{s}^{t} \mathrm{E}_{\mu \times \mathrm{P}}\left(\left\|a_{z}^{m}-a_{z}\right\|_{H}^{2} \rho_{s, z}^{m}\right) d z+2 \int_{s}^{t}\left\|D a_{z}\right\|_{\infty}^{2} \mathrm{E}_{\mu \times \mathrm{P}}\left\|\varphi_{s, z}^{m}-\varphi_{s, z}\right\|_{H}^{2} d z
\end{aligned}
$$

Первое слагаемое в правой части (6.7) сходится к нулю равномерно по $s, t$ благодаря (6.4), равномерной ограниченности $\left\{a_{t}^{m}, a_{t} ; t \in[0, T], m \geqslant 1\right\}$ и равномерной интегрируемости $\left\{\rho_{s, z}^{m} ; m \geqslant 1, s, z \in[0, T]\right\}$. Второй член в правой части (6.6) рассматривается аналогично.

Таким образом, имеет место неравенство

$$
\mathrm{E}_{\mu \times \mathrm{P}}\left\|\varphi_{s, t}^{m}-\varphi_{s, t}\right\|_{H}^{2} \leqslant \varepsilon_{m}+2\left(A_{1}^{2}+S_{1}\right) \int_{s}^{t} \mathrm{E}_{\mu \times \mathrm{P}}\left\|\varphi_{s, z}^{m}-\varphi_{s, z}\right\|_{H}^{2} d z
$$

где $\varepsilon_{m} \rightarrow 0, m \rightarrow \infty$.

Сходимость (6.5) следует из леммы Гронуолла.

По лемме 4.1 и $(6.5)$ мера $(\mu \times \mathrm{P}) \circ\left(\varphi_{s, t}\right)^{-1}$ абсолютно непрерывна относительно $\mu \times \mathrm{P}$. Поэтому для всех $s \leqslant t$ и для п.в. $\omega \in \Omega$

$$
\mu \circ\left(\varphi_{s, t}(\omega, \cdot)\right)^{-1} \ll \mu
$$

ЗАмечАнИЕ. Соответствующее множество Р-нулевой меры зависит, вообще говоря, от $s$ и $t$.

Для того чтобы доказать формулы (3.2), (3.3) и утверждение b) теоремы 3.1, нам потребуется следующая несложная лемма о пределе суперпозиции функций (см., например, [6]).

Лемма 6.2. Пусть $X, Y$ - полные сепарабельные метрические пространства, $\mu$ - вероятностная мера на $X, \varphi_{n}: X \rightarrow X, f_{n}: X \rightarrow Y, n \geqslant 0$,измеримые функиии.

Предположим, что

(i) $\varphi_{n} \stackrel{\mu}{\longrightarrow} \varphi_{0}, n \rightarrow \infty ; f_{n} \stackrel{\mu}{\longrightarrow} f_{0}, n \rightarrow \infty$;

(ii) для любого $n \geqslant 1$ образ меры $\mu \circ \varphi_{n}^{-1}$ абсолютно непрерывен относительно $\mu$; (iii) последовательность плотностей $\left\{\frac{d \mu \circ \varphi_{n}^{-1}}{d \mu}, n \geqslant 1\right\}$ равномерно ин-
тегрируема. 
Тогда $f_{n} \circ \varphi_{n} \stackrel{\mu}{\longrightarrow} f_{0} \circ \varphi_{0}, n \rightarrow \infty$.

Рассмотрим обратное стохастическое уравнение с обращенньм винеровским процессом $\widetilde{\omega}_{n}(t)=\omega_{n}(T)-\omega_{n}(t)$. Тогда аналогично предыдущим рассуждениям можно доказать существование измеримого "обратного потока" $\varphi_{t, s}, s \leqslant t$, и абсолютную непрерывность $\mu \circ\left(\varphi_{t, s}\right)^{-1} \ll \mu$ для п.в. $\omega$.

Применяя лемму 6.2 и свойство потока для стохастических дифференциальных уравнений в конечномерном пространстве, получаем

$$
\begin{gathered}
\forall t_{1}, t_{2}, t_{3} \in[0, T] \exists \Omega_{t_{1}, t_{2}, t_{3}}, \quad \mathrm{P}\left(\Omega_{t_{1}, t_{2}, t_{3}}\right)=1, \quad \forall \omega \in \Omega_{t_{1}, t_{2}, t_{3}} \\
\varphi_{t_{2}, t_{3}} \circ \varphi_{t_{1}, t_{2}}=\varphi_{t_{1}, t_{3}} \quad \mu \text {-п.н. }
\end{gathered}
$$

Для доказательства (3.2), (3.3) нам потребуется бесконечномерная версия лемм 4.3 и 4.4.

Лемма 6.3. Для всех $s, t \in[0, T], s \leqslant t, u$ n.в. $\omega$

а) для любого $p \geqslant 1$ отображение $u \mapsto\left(\varphi_{s, t}(u, \omega)-u\right)$ принадлежит пространству $W_{p}^{2}(H)$,

b) для производныл

$$
D \varphi_{s, t}(u):=\operatorname{id}_{H}+D\left(\varphi_{s, t}(u)-u\right)
$$

выполняются все оценки леммы 4.4 с теми жее константами,

c) производная $D \varphi_{s, t}$ удовлетворяет следующему соотношению:

$$
\begin{aligned}
D \varphi_{s, t}=\operatorname{id}_{H} & +\int_{s}^{t} D a_{z}\left(\varphi_{s, z}\right) D \varphi_{s, z} d z \\
& +\sum_{n} \int_{s}^{t} D \sigma_{z}^{n}\left(\varphi_{s, z}\right) D \varphi_{s, z} d \omega_{n}(z)
\end{aligned}
$$

для п.в. $(\omega, u) \in \Omega \times \mathbb{R}^{\infty}$.

ДокАЗАТЕЛьство. Заметим, что по лемме 4.4

$$
\left(\varphi_{s, t}^{m}(u)-u\right) \in W_{p}^{2}(H) \text { для п.в. } \omega,
$$

причем $\pi_{m} D\left(\varphi_{s, t}^{m}(u)-u\right)=D\left(\varphi_{s, t}^{m}(u)-u\right)$, потому что функция $u \mapsto \varphi_{s, t}^{m}(u, \omega)-u$ (как функция от $u \in X$ при фиксированном $\omega$ ) зависит только от $u_{m}$. Для простоты предположим, что $s=0$. Обозначим $\varphi_{t}:=\varphi_{0, t}$. Докажем фундаментальность последовательности $\left\{D\left(\varphi_{t}^{m}(u)-u\right), m \geqslant 1\right\}$. Применим формулу Ито к $\left\|D \varphi_{t}^{m}-D \varphi_{t}^{k}\right\|_{H S}^{p}$ :

$$
\begin{aligned}
\mathrm{E}_{\mu \times \mathrm{P}} \| & D \varphi_{t}^{m}-D \varphi_{t}^{k} \|_{H S}^{p} \\
\leqslant & K . \mathrm{E}_{\mu \times \mathrm{P}}\left\{\int_{0}^{t}\left\|D \varphi_{s}^{m}-D \varphi_{s}^{k}\right\|^{p-1}\left\|\left(D a_{s}^{m}\right)\left(\varphi_{s}^{m}\right) D \varphi_{s}^{m}-\left(D a_{s}^{k}\right)\left(\varphi_{s}^{k}\right) D \varphi_{s}^{k}\right\|\right. \\
& \left.\quad+\sum_{n}\left\|D \varphi_{s}^{m}-D \varphi_{s}^{k}\right\|^{p-2}\left\|D \sigma_{s}^{m, n}\left(\varphi_{s}^{m}\right) D \varphi_{s}^{m}-D \sigma_{s}^{k, n}\left(\varphi_{s}^{k}\right) D \varphi_{s}^{k}\right\|^{2}\right\} d s \\
\leqslant & K . \mathrm{E}_{\mu \times \mathrm{P}} \int_{0}^{t}\left\|D \varphi_{s}^{m}-D \varphi_{s}^{k}\right\|^{p} d s \\
& \quad+K . \mathrm{E}_{\mu \times \mathrm{P}} \int_{0}^{t}\left(\left\|D a_{s}^{m}\left(\varphi_{s}^{m}\right)-D a_{s}^{k}\left(\varphi_{s}^{k}\right)\right\|\right. \\
& \left.+\sum_{n}\left\|D \sigma_{s}^{m, n}\left(\varphi_{s}^{m}\right)-D \sigma^{k, n}\left(\varphi_{s}^{k}\right)\right\|\right) \xi_{k, m}(s) d s \\
= & I_{1}(m, k)+I_{2}(m, k),
\end{aligned}
$$


где $K .=K .(p, T)$ - некоторая константа, $\xi_{k, m}(s), s \in[0, T],-$ некоторый положительный процесс (см. лемму 4.4) такой, что $\sup _{s, k, m} \mathrm{E}_{\mu \times \mathrm{P}}\left(\xi_{k, m}(s)\right)^{p}<\infty$ для любого $p>0$.

По лемме 6.2 и теореме Лебега о мажорируемой сходимости $I_{2}(m, k) \rightarrow 0$ при $m, k \rightarrow \infty$.

Применяя лемму Гронуолла к (6.10), получаем

$$
\mathrm{E}_{\mu \times \mathrm{P}}\left\|D \varphi_{t}^{m}-D \varphi_{t}^{n}\right\|_{H S}^{p} \rightarrow 0, \quad m, n \rightarrow \infty .
$$

Обозначим предел $D \varphi_{t}^{m}$ через $\psi_{t}$. Также уже было доказано, что

$$
\mathrm{E}_{\mu \times \mathrm{P}}\left\|\varphi_{t}^{m}-\varphi_{t}\right\|^{p} \rightarrow 0, \quad m \rightarrow \infty .
$$

Выберем такую подпоследовательность $\left\{m_{k}\right\}$, что

$$
\mathrm{E}_{\mu}\left\|\varphi_{t}^{m_{k}}-\varphi_{t}\right\|^{p} \rightarrow 0, \quad m_{k} \rightarrow \infty
$$

и

$$
\mathrm{E}_{\mu}\left\|D \varphi_{t}^{m_{k}}-\psi_{t}\right\|^{p} \rightarrow 0, \quad m_{k} \rightarrow \infty
$$

для всех $\omega \in \Omega_{t}$, где $\Omega_{t}$ - некоторое множество полной меры Р. Тогда $\psi_{t}=D \varphi_{t}$, отображение $u \mapsto\left(\varphi_{t}(u)-u\right)$ принадлежит $W_{p}^{1}(H)$, и утверждение b) доказано. Для доказательства с) заметим, что $D \varphi_{s, t}^{m}$ удовлетворяет уравнению $(6.9)$ с функциями $a^{m}, \sigma^{m, n}$ вместо $a, \sigma^{n}$ соответственно. Переход к пределу при $m \rightarrow \infty$ и применение лемм 6.2 и $6.3 \mathrm{a})$, b) завершает доказательство утверждения с). Производные второго порядка рассматриваются аналогично.

Лемма 6.4. Предположим, что $b: X \rightarrow H$ принадлежст пространству $W_{\infty}^{1}(H)$. Тогда формула (4.9) справедлива для $\mu \times \mathrm{P}_{-}$почти всех $(u, \omega)$, если заменить оператор $\nabla$ на $D$.

ЗАмечАНИЕ. Здесь мы считаем, что $D \varphi_{s}(u):=\operatorname{id}_{H}+D\left(\varphi_{s}(u)-u\right)$. Отметим, что $D \varphi_{s}$ не является оператором Гильберта-Шмидта, но $\left(D \sigma_{z}^{n}\right)\left(\varphi_{z}\right) D \varphi_{z}$ является таковым, и поэтому стохастические интегралы и след корректно определены, так как

$$
\sum_{n} \int_{0}^{T} \mathrm{E}_{\mu \times \mathrm{P}}\left\|\nabla \sigma_{z}^{n}\left(\varphi_{z}\right) \nabla \varphi_{z}\right\|_{H S}^{2} d z<\infty .
$$

ДокАЗАТЕЛЬСтво. Пусть $\left\{b_{m}: X \rightarrow H, m \geqslant 1\right\}$ - последовательность таких функций, что $\forall u \quad b_{m}(u)=\pi_{m} b_{m}\left(\pi_{m} u\right)$,

$$
\sup _{m, u}\left\|b_{m}(u)\right\| \leqslant \underset{u}{\operatorname{ess} \sup }\|b(u)\|, \quad \sup _{m, u}\left\|D b_{m}(u)\right\| \leqslant \underset{u}{\operatorname{ess} \sup \|D b(u)\|}
$$

и для $\mu$-п.в. $u \in X \quad b_{m}(u) \rightarrow b(u)$ и $D b_{m}(u) \rightarrow D b(u)$ при $m \rightarrow \infty$. Данная последовательность может быть выбрана аналогично выбору $\left\{a^{m, n, k_{m}}\right\}$ в начале доказательства теоремы 4.1.

Из лемм 6.2 и 6.3 следует сходимость

$$
\begin{gathered}
b_{m}\left(\varphi_{s}^{m}\right) \rightarrow b\left(\varphi_{s}\right), \quad m \rightarrow \infty \\
D\left(b_{m}\left(\varphi_{s}^{m}\right)\right)=\left(D b_{m}\right)\left(\varphi_{s}^{m}\right) D \varphi_{s}^{m} \rightarrow(D b)\left(\varphi_{s}\right) D \varphi_{s}, \quad m \rightarrow \infty
\end{gathered}
$$

в любом пространстве $L_{p}, p \geqslant 1$. В частности, имеет место сходимость

$$
b_{m} \rightarrow b, \quad m \rightarrow \infty, \quad \text { в } W_{2}^{1}(H)
$$


и

$$
b_{m}\left(\varphi_{s}^{m}\right) \rightarrow b\left(\varphi_{s}\right), \quad m \rightarrow \infty, \quad \text { в } L_{2}\left(\Omega, \mathrm{P}, W_{2}^{1}(X, \mu)\right)
$$

Поэтому $\left(\delta b^{m}\right)\left(\varphi_{s}^{m}\right) \rightarrow(\delta b)\left(\varphi_{s}\right)$ по мере $\mu \times \mathrm{P}_{\text {и }}$

$$
\delta\left(b^{m}\left(\varphi_{s}^{m}\right)\right) \rightarrow \delta\left(b\left(\varphi_{s}\right)\right) \quad \text { в } L_{2}(X \times \Omega, \mu \times \mathrm{P}) .
$$

Применяя леммы 6.2 и 6.3 к остальным членам (4.9), мы завершаем доказательство леммы 6.4.

Докажем (3.2) для всех $\omega \in \Omega_{s, t}$, где $\Omega_{s, t}-$ некоторое множество полной Р-меры. Для этого достаточно проверить (лемма 4.1), что

$$
\mathrm{E}_{\mu \times \mathrm{P}}\left(\ln \rho_{s, t}^{m}-\ln \rho_{s, t}\right)^{2} \rightarrow 0, \quad m \rightarrow \infty,
$$

где $\rho_{s, t}$ задано форомулой (3.2).

Для простоты предположим, что $t=0$. Обозначим $\varphi_{s}:=\varphi_{0, s}$. Тогда

$$
\begin{aligned}
\mathrm{E}_{\mu \times \mathrm{P}}( & \left.\ln \rho_{s, 0}^{m}-\ln \rho_{s, 0}\right)^{2} \\
\leqslant & 4 \mathrm{E}_{\mu \times \mathrm{P}} \int_{0}^{t}\left\{\left(\left(\delta a_{s}\right)\left(\varphi_{s}\right)-\left(\delta a_{s}^{m}\right)\left(\varphi_{s}^{m}\right)\right)^{2}\right. \\
& +\sum_{n}\left(\delta\left(D_{\sigma_{s}^{n}} \sigma_{s}^{n}\right)\left(\varphi_{s}\right)-\delta\left(D_{\left.\left.\sigma_{s}^{m, n} \sigma_{s}^{m, n}\right)\left(\varphi_{s}^{m}\right)\right)^{2}}\right.\right. \\
& +\sum_{n}\left(\left(\delta \sigma_{s}^{n}\right)\left(\varphi_{s}\right)-\left(\delta \sigma_{s}^{m, n}\right)\left(\varphi_{s}^{m}\right)\right)^{2} \\
& \left.+\sum_{n}\left(\left(D_{\sigma_{s}^{n}}\left(\delta \sigma_{s}^{n}\right)\right)\left(\varphi_{s}\right)-\left(D_{\sigma_{s}^{m, n}}\left(\delta \sigma_{s}^{m, n}\right)\right)\left(\varphi_{s}^{m}\right)\right)^{2}\right\} d s
\end{aligned}
$$

Сходимость правой части (6.13) к нулю при $m \rightarrow \infty$ может быть проверена при помощи $(6.5),(6.11)$ и лемм 2.1, 6.2-6.4.

Формула (3.3) для $s<t$ следует из (4.6), (3.2) и (6.8).

Проверим, что утверждения b)-d) теоремы 3.1 выполняются на некотором общем множестве $\Omega_{0}, \mathrm{P}\left(\Omega_{0}\right)=1$, не зависящем от $t_{1}, t_{2}, t_{3}, s, t$.

Для этого достаточно доказать, что сушествуют модификация функции $\rho_{s, t}$ и множество $\Omega_{1}, \mathrm{P}\left(\Omega_{1}\right)=1$, такие, что для каждого $\omega \in \Omega$ совокупность функций $\left\{\rho_{s, t}(\cdot, \omega), s, t \in[0, T]\right\}$, заданных в $(3.2),(3.3)$, равномерно интегрируема относительно меры $\mu$ и $\rho_{s, t}(\cdot, \omega)$ непрерывно по $s, t$ в топологии сходимости по мере $\mu$. Действительно, допустим, это выполняется. Напомним, что $\varphi_{s, t}(u, \omega)$ непрерьвно по $s, t$ для всех $\omega \in \Omega, u \in X$. Пусть $\Omega_{2}$ - такое множество, что $\rho_{s, t}(\cdot, \omega)$, заданное в $(3.2)$, совпадает с плотностью Радона-Никодима $\frac{d \mu \circ \varphi_{s, t}^{-1}}{d \mu}$ для всех рациональных $s, t$. Отметим, что $\mathrm{P}\left(\Omega_{2}\right)=1$. Тогда по лемме $4.1 \rho_{s, t}$ - искомая плотность Радона-Никодима для всех $s, t \in[0, T], \omega \in \Omega_{0}=\Omega_{1} \cap \Omega_{2}$. Утверждения b), с) теоремы 3.1 выполнены для п.в. $\omega$ для рациональных $t_{1}, t_{2}, t_{3}, s, t$. Значит, они справедливы для всех $t_{1}, t_{2}, t_{3}, s, t$ по лемме 6.2 .

Для того чтобы доказать сушествование модификации $\left\{\rho_{s, t}(\cdot, \omega), 0 \leqslant s \leqslant t\right\}$, которая равномерно интегрируема относительно $\mu$ для п.в. $\omega$, достаточно проверить сушествование непрерывной по $s, t, s \leqslant t$, версии

$$
\mathrm{E}_{\mu}\left|\rho_{s, t} \ln \rho_{s, t}\right|=\mathrm{E}_{\mu}\left|\ln \rho_{t, s}\right|=\mathrm{E}_{\mu}\left|\int_{s}^{t}\left(\delta \widetilde{a}_{z}\right)\left(\varphi_{s, z}\right) d z+\sum_{n} \int_{s}^{t} \delta \sigma_{z}^{n}\left(\varphi_{s, z}\right) \circ d \omega_{n}(z)\right| .
$$


Применим теорему Колмогорова. Допустим, для удобства, что $0 \leqslant s \leqslant s_{1} \leqslant t_{1} \leqslant$ $t \leqslant T$. Тогда

$$
\begin{aligned}
& \mathrm{E}_{p}\left(\mathrm{E}_{\mu}\left|\rho_{s, t} \ln \rho_{s, t}\right|-\mathrm{E}_{\mu}\left|\rho_{s_{1}, t_{1}} \ln \rho_{s_{1}, t_{1}}\right|\right)^{6} \\
& \leqslant 10 \sup _{z \geqslant s} \mathrm{E}_{\mu \times \mathrm{P}}\left\{\left(\left(\delta \widetilde{a}_{z}\right)\left(\varphi_{s, z}\right)\right)^{6}+\left(\sum_{n} D_{\sigma_{z}^{n}}\left(\delta \sigma_{z}^{n}\right)\left(\varphi_{s, z}\right)\right)^{6}\right\}\left(\left|s-s_{1}\right|^{6}+\left|t-t_{1}\right|^{6}\right) \\
& \quad+\sup _{s_{1} \leqslant z \leqslant t_{1}} \mathrm{E}_{\mu \times \mathrm{P}}\left(\left(\delta a_{z}\right)\left(\varphi_{s, z}\right)-\left(\delta a_{z}\right)\left(\varphi_{s_{1}, z}\right)\right)^{6} \\
& \quad+\sup _{s_{1} \leqslant z \leqslant t_{1}} \mathrm{E}_{\mu \times \mathrm{P}}\left(\sum_{n}\left(\left(\delta \sigma_{z}^{n}\right)\left(\varphi_{s, z}\right)-\left(\delta \sigma_{z}^{n}\right)\left(\varphi_{s_{1}, z}\right)\right)^{2}\right)^{3} \\
& =I_{1}+I_{2}+I_{3} .
\end{aligned}
$$

Ограниченность супремума математического ожидания в $I_{1}$ проводится аналогично доказательству теоремы 4.1. Рассуждения для $I_{2}$ и $I_{3}$ почти одинаковые. Ограничимся рассмотрением $I_{2}$. Применим лемму 6.4:

$$
\begin{aligned}
\mathrm{E}_{\mu \times \mathrm{P}}( & \left.\left(\delta a_{z}\right)\left(\varphi_{s, z}\right)-\left(\delta a_{z}\right)\left(\varphi_{s_{1}, z}\right)\right)^{6} \\
\leqslant & 3\left\{\mathrm{E}_{\mu \times \mathrm{P}}\left[\delta\left(a_{z}\left(\varphi_{s, z}\right)-a_{z}\left(\varphi_{s_{1}, z}\right)\right)\right]^{6}\right. \\
& +\mathrm{E}_{\mu \times \mathrm{P}}\left[\left\langle a_{z}\left(\varphi_{s, z}\right), \int_{s}^{z} a_{\tau}\left(\varphi_{s, \tau}\right) d \tau+\sum_{n} \int_{s}^{z} \sigma_{\tau}^{n}\left(\varphi_{s, \tau}\right) d \omega_{n}(\tau)\right\rangle\right. \\
& \left.-\left\langle a_{z}\left(\varphi_{s_{1}, z}\right), \int_{s_{1}}^{z} a_{\tau}\left(\varphi_{s_{1}, \tau}\right) d \tau+\sum_{n} \int_{s_{1}}^{z} \sigma_{\tau}^{n}\left(\varphi_{s_{1}, \tau}\right) d \omega_{n}(\tau)\right\rangle\right]^{6} \\
& +\mathrm{E}_{\mu \times \mathrm{P}}\left[\operatorname { t r } \left(( D a _ { z } ) ( \varphi _ { s , z } ) \left\{\int_{s}^{z}\left(D a_{\tau}\right)\left(\varphi_{s, \tau}\right) D \varphi_{s, \tau} d \tau\right.\right.\right. \\
& \left.+\sum_{n} \int_{s}^{z}\left(D \sigma_{\tau}^{n}\right)\left(\varphi_{s, \tau}\right) D \varphi_{s, \tau} d \omega_{n}(\tau)\right\} \\
& -\left(D a_{z}\right)\left(\varphi_{s_{1}, z}\right)\left\{\int_{s_{1}}^{z}\left(D a_{\tau}\right)\left(\varphi_{s_{1}, \tau}\right) D \varphi_{s_{1}, \tau} d \tau\right. \\
& \left.\left.\left.\left.+\sum_{n} \int_{s}^{z}\left(D \sigma_{\tau}^{n}\right)\left(\varphi_{s_{1}, \tau}\right) D \varphi_{s_{1}, \tau} d \omega_{n}(\tau)\right\}\right)\right]^{6}\right\}
\end{aligned}
$$

Лемма 6.5. Для кажсдого $p \geqslant 2$ существует константа $K_{p}$ такая, что $\forall j \in\{0,1,2\}, \forall s, s_{1}, z, s<s_{1}<z$,

$$
\mathrm{E}_{\mu \times \mathrm{P}}\left\|D^{j} \varphi_{s, z}-D^{j} \varphi_{s_{1}, z}\right\|^{p} \leqslant K_{p}\left|s-s_{1}\right|^{p / 2}
$$

Доказательство проводится таким же образом, как и в конечномерном случае (см., например, [1]).

Используя лемму 6.5 для правой части (6.14) и лемму 2.1 для оценки моментов дивергенции, получаем следующее неравенство:

$\mathrm{E}_{\mathrm{P}}\left(\mathrm{E}_{\mu}\left|\rho_{s, t} \ln \rho_{s, t}\right|-\mathrm{E}_{\mu}\left|\rho_{s_{1}, t_{1}} \ln \rho_{s_{1}, t_{1}}\right|\right)^{6} \leqslant C\left(\left|s-s_{1}\right|^{3}+\left|t-t_{1}\right|^{3}\right), \quad s \leqslant t, s_{1} \leqslant t_{1}$.

Значит, по теореме Колмогорова $\mathrm{E}_{\mu}\left|\rho_{s, t} \ln \rho_{s, t}\right|$ непрерывно по $(s, t)$ и, следовательно, $\sup _{s \leqslant t} \mathrm{E}_{\mu}\left|\rho_{s, t} \ln \rho_{s, t}\right|<\infty$ для п.в. $\omega$. Поэтому для п.в. $\omega$ и всех $s \leqslant t$ имеем абсолютную непрерывность $\mu \circ \varphi_{s, t}^{-1} \ll \mu$. 
Аналогично, рассматривая обрашенное стохастическое уравнение, получаем $\mu \circ \varphi_{t, s}^{-1} \ll \mu$ для $s \leqslant t$, а также равномерную интегрируемость $\left\{\frac{d \mu \circ \varphi_{t, s}(\cdot, \omega)^{-1}}{d \mu}\right.$, $s \leqslant t\}$ для п.в. $\omega$. Таким образом, утверждение b) теоремы 3.1 истинно. Для доказательства (3.2) и (3.3) для п.в. $\omega$ и всех $s, t$ достаточно заметить, что для п.в. $\omega$ функция $\left\{\rho_{t, s}(\cdot, \omega), s \leqslant t\right\}$ непрерывна по $s, t$ в топологии сходимости по мере $\mu$.

Теорема 3.1 доказана.

\section{Список литературы}

1. Kunita $H$. Stochastic flows and stochastic differential equations. Cambridge: Cambridge Univ. Press, 1990. (Cambridge Stud. Adv. Math. V. 24.)

2. Скороход A. В. Случайные линейные операторы. Киев: Наукова думка, 1978.

3. Cruzeiro A.-B. Equations différentielles sur l'espace de Wiener et formules de CameronMartin non-linéaires // J. Funct. Anal. 1983. V. 54. P. 206-227.

4. Bogachev V. I., Mayer-Wolf E. Absolutely continuous flows generated by Sobolev class vector fields in finite and infinite dimensions // J. Funct. Anal. 1999. V. 167. P. 1-68.

5. Ustunel A.S., Zakai M. Transformations of Wiener measure under anticipative flows // Probab. Theory Related Fields. 1992. V. 93. P. 91-136.

6. Кулик A. М., Пилипенко А. Ю. Нелинейные преобразования гладких мер в бесконечномерном пространстве // Укр. матем. журн. 2000. Т. 52. № 9. С. 1403-1431.

7. Watanabe $S$. Lectures on stochastic differential equations and the Malliavin calculus. Berlin: Springer-Verlag, 1984.

8. Богачев В.И. Гауссовские меры. М.: Наука, 1997.

9. Гихман И.И., Скороход А.В. О плотностях вероятностных мер в функциональных пространствах // УМН. 1966. Т. 21. №6. С. 83-152.

10. Stricker C., Yor M. Calcul stochastique dependant d'un paramètre // Z. Wahrsch. Verw. Gebiete. 1978. V. 45. № 2. P. 109-133.

Институт математики НАН Украины, Киев, Украина

E-mail : apilip@imath.kiev.ua

Поступила в редакцию

23.05 .2002 